\title{
Hadron masses and decay constants with Wilson quarks at $\beta=5.85$ and 6.0
}

\author{
Y. Iwasaki, ${ }^{1,2}$ K. Kanaya, ${ }^{1,2}$ T. Yoshié, ${ }^{1,2}$ T. Hoshino, ${ }^{3}$ T. Shirakawa, ${ }^{3}$ Y. Oyanagi, ${ }^{4}$ S. Ichii, ${ }^{5}$ and T. Kawai ${ }^{6}$ \\ (QCDPAX Collaboration) \\ ${ }^{1}$ Institute of Physics, University of Tsukuba, Ibaraki 305, Japan \\ ${ }^{2}$ Center for Computational Physics, University of Tsukuba, Ibaraki 305, Japan \\ ${ }^{3}$ Institute of Engineering Mechanics, University of Tsukuba, Ibaraki 305, Japan \\ ${ }^{4}$ Department of Information Science, University of Tokyo, Tokyo 113, Japan \\ ${ }^{5}$ Computer Centre, University of Tokyo, Tokyo 113, Japan \\ ${ }^{6}$ Department of Physics, Keio University, Yokohama 223, Japan
}

(Received 29 December 1995)

\begin{abstract}
We present results of a high statistics calculation of hadron masses and meson decay constants in the quenched approximation to lattice QCD with Wilson quarks at $\beta=5.85$ and 6.0 on $24^{3} \times 54$ lattices. We analyze the data paying attention in particular to the systematic errors due to the choice of fitting range and due to the contamination from excited states. We find that the systematic errors for the hadron masses with quarks lighter than the strange quark amount to 1 to 2 times the statistical errors. When the lattice scale is fixed from the $\rho$ meson mass, the masses of the $\Omega^{-}$baryon and the $\phi$ meson at two $\beta$ 's agree with experiment within about one standard deviation. On the other hand, the central value of the nucleon mass at $\beta=6.0(5.85)$ is larger than its experimental value by about $15 \%(20 \%)$ and that of the $\Delta$ mass by about $15 \%$ (4\%): Even when the systematic errors are included, the baryon masses at $\beta=6.0$ do not agree with experiment. Vector meson decay constants at two values of $\beta$ agree well with each other and are consistent with experiment for a wide range of the quark mass, when we use current renormalization constants determined nonperturbatively by numerical simulations. The pion decay constant agrees with experiment albeit with large errors. Results for the masses of excited states of the $\rho$ meson and the nucleon are also presented. [S0556-2821(96)00711-4]
\end{abstract}

PACS number(s): 11.15.Ha, 12.38.Gc

\section{INTRODUCTION}

Although there have been many efforts to calculate hadron masses in lattice QCD by numerical simulations, it has turned out that the derivation of convincing results is much harder than thought at the beginning, even in the quenched approximation. For example, before 1988, there was a large discrepancy among the results for the mass ratio $m_{N} / m_{\rho}$ obtained for $\beta=6 / g^{2}=5.7-6.0$ and in the quark mass region corresponding to $m_{\pi} / m_{\rho} \geqslant 0.5$. The discrepancy was caused by systematic errors due to contamination from excited states $[1,2]$ and effects of finite lattice spacing [3] and finite lattice volume. Recent high statistics simulations employ lattices with a large temporal extent [4-6] and/or extended quark sources [5-11] to reduce fluctuations as well as the contamination from excited states. However, a long plateau in an effective mass is rarely seen and data for effective masses frequently show large fluctuations at large time separations. The uncertainty in the choice of fitting range is, therefore, another source of systematic errors. In order to obtain reliable values for the spectrum, it is essential to make a quantitative study of these systematic errors.

In this paper we report results of a high statistics calculation of the quenched QCD spectrum with the Wilson quark action at $\beta=5.85$ and 6.0 on $24^{3} \times 54$ lattices. Our major objective is to calculate light hadron masses as well as meson decay constants, paying attention, in particular, to the systematic errors due to the choice of fitting range and due to the contamination from excited states. In order to estimate the magnitude of these systematic errors, we perform corre- lated one-mass fits to hadron propagators, systematically varying fitting ranges $[5,12]$. Assuming the ground state dominance at large time separations, we estimate systematic errors in hadron masses which cannot be properly taken into account by the standard least mean square fit when the fitting range is fixed. It is shown that, for the hadron masses with quarks lighter than the strange quark, the systematic errors amount to 1-2 times the statistical errors. We then perform correlated two-mass fits, again varying fitting ranges. We find that the ground state mass is consistent with that obtained from the one-mass fit within the statistical and systematic errors. Finally, we extrapolate the results of hadron masses at finite quark mass to the chiral limit, taking account of systematic errors both due to the choice of extrapolation function and due to the fitting range. We also study meson decay constants in a similar way.

We use a point source in this study. Historically there was a report that numerical results for hadron masses appear to depend on the type of the source adopted [13], although it was afterward reported in some works that the masses are independent within statistical errors $[5,6]$. Note in this connection that there is no proof that the value of a hadron mass is independent of the type of source in the case of the quenched approximation, due to the lack of the transfer matrix, and that there is the so-called Gribov problem for gauge fixing which is necessary for almost all smeared sources. Under these circumstances it may be worthwhile to present the details of the results and the analysis with the point source as a reference. The method of analysis of the systematic errors in this work can be applied to the cases of smeared sources too. 
TABLE I. Hopping parameters and average number of iterations used to solve quark propagators. Approximate values for $m_{\pi} / m_{\rho}$ are also given. Table VIII contains precise values for $m_{\pi} / m_{\rho}$.

\begin{tabular}{ccccc}
\hline \hline \multicolumn{2}{c}{$\beta=5.85$} & \multicolumn{3}{c}{$\beta=6.0$} \\
$K$ & No. iteration & $K$ & No. iteration & $m_{\pi} / m_{\rho}$ \\
\hline 0.1440 & $80 \pm 3$ & 0.1450 & $90 \pm 3$ & 0.97 \\
0.1540 & $160 \pm 10$ & 0.1520 & $160 \pm 10$ & 0.87 \\
0.1585 & $420 \pm 45$ & 0.1550 & $380 \pm 40$ & 0.70 \\
0.1595 & $610 \pm 75$ & 0.1555 & $430 \pm 45$ & 0.64 \\
0.1605 & $1850 \pm 410$ & 0.1563 & $1110 \pm 170$ & 0.52 \\
\hline \hline
\end{tabular}

Numerical simulations are performed with the QCDPAX [14], a MIMD parallel computer constructed at the University of Tsukuba. For the calculations performed in this work, we use $24 \times 18$ processing units interconnected in a toroidal two-dimensional mesh with a peak speed of 12.4 GFLOPS. (The maximum number of nodes is $24 \times 20$ with a peak speed of 14.0 GFLOPS.) The sustained speed for the Wilson quark matrix multiplication is approximately 5 GFLOPS. The calculations described here took about six months on the QCDPAX.

We start by giving in Sec. II some details about our numerical simulations. Then we derive hadron masses at finite quark mass in Sec. III and perform two-mass fits to estimate the masses of excited states of the $\rho$ meson and the nucleon in Sec. IV. We extrapolate the results to the chiral limit in Sec. V. Section VI is devoted to the evaluation of meson decay constants. In Sec. VII, we give conclusions and discussion of the results.

\section{NUMERICAL CALCULATION}

We use the standard one-plaquette gauge action

$$
S_{g}=\frac{2}{g^{2}} \sum_{P} \operatorname{Re} \operatorname{Tr}\left(U_{P}\right)
$$

and the Wilson quark action

$$
\begin{gathered}
S_{q}=-\sum_{n, m} \bar{\psi}(n) D(K, n, m) \psi(m), \\
D(K, n, m)=\delta_{n, m}-K \sum_{\mu}\left\{\left(I-\gamma_{\mu}\right) U_{n, \mu} \delta_{n+\mu, m}\right. \\
\left.+\left(I+\gamma_{\mu}\right) U_{m, \mu}^{\dagger} \delta_{m+\mu, n}\right\},
\end{gathered}
$$

where $g$ is the bare coupling constant and $K$ is the hopping parameter.

Simulations are done on $24^{3} \times 54$ lattices at $\beta=6 / g^{2}=5.85$ and 6.0 for the five values of the hopping parameter listed in Table I. The mass ratio $m_{\pi} / m_{\rho}$ takes a value from 0.97 to 0.52 and the values roughly agree with each other at two $\beta$ 's for the five cases of the hopping parameter. We choose the values of the third largest hopping parameter in such a way that they approximately correspond to the strange quark.

We generate 100 (200) configurations with periodic boundary conditions at $\beta=5.85$ (6.0) by a Cabibbo-
Marinari-Okawa algorithm with an eight-hit pseudo-heatbath algorithm for three SU(2) subgroups. The acceptance rate is about 0.95 for both $\beta$ 's. Each configuration is separated by 1000 sweeps after a thermalization of $6000(22000)$ sweeps at $\beta=5.85$ (6.0).

The quark propagator $G$ on a configuration given by

$$
\sum_{m} D(K, n, m) G(m)=B(n)
$$

is constructed using a red-black minimal residual algorithm, taking periodic boundary conditions in all directions. We employ the point source at the origin $B(n)=\delta_{n, 0}$.

The convergence criterion we take for the quark matrix inversion is that both of the following two conditions be satisfied:

$$
\begin{gathered}
\sqrt{|R|^{2} /(3 \times 4 \times V)}<10^{-9}, \\
\max _{n, c, s}\left\{\left|R_{c, s}(n) / G_{c, s}(n)\right|\right\}<0.03,
\end{gathered}
$$

where $|R|$ is the norm of the residual vector $R=B-D(K) G, V=L^{3} \times T$ is the lattice volume ( $L=24$ is the lattice size in the spatial directions and $T=54$ is that in the temporal direction), and $c$ and $s$ are color and spin indices. The average number of iterations needed for the convergence is given in Table I.

Selecting several configurations, we have solved Eq. (4) exactly within single precision to construct an exact hadron propagator and compared it with that obtained with the stopping conditions above. We find that the difference in a hadron propagator (for any particle at any time slice) is at most $1 \%$ of the statistical error estimated using all (100 or 200) configurations. Therefore the error due to truncation of iterations is small enough and does not affect the following analyses and results.

We use $\bar{u} \Gamma d$ for meson operators with $\Gamma=\gamma_{5}$ for $\pi$, $i \gamma_{0} \gamma_{5}$ for $\pi(\widetilde{\pi})$, and $\gamma_{i}$ for $\rho$. For baryons, we use nonrelativistic operators

$$
\begin{gathered}
N_{l}=\epsilon^{a b c} \sum_{i, j}^{2} u_{i}^{a} \tau_{3}^{i j} d_{j}^{b} u_{l}^{c}, \quad l=1,2, \\
\Delta_{l}=\epsilon^{a b c} \sum_{i, j, k}^{2} S_{l}^{i j k} u_{i}^{a} u_{j}^{b} u_{k}^{c}, \quad l= \pm 3 / 2, \pm 1 / 2,
\end{gathered}
$$

where $\tau_{3}$ is the third component of Pauli matrices and $S_{l}$ is the projection operator to the $J=3 / 2, J_{z}=l$ state. We also use antibaryon operators obtained by replacing the upper components of the Dirac spinor in Eqs. (7) and (8) with the lower components.

We average zero momentum hadron propagators over all states with the same quantum numbers: three polarization states for the $\rho$ meson and two (four) spin states for the nucleon $(\Delta)$. Then we average the propagators for the particle and the antiparticle: For mesons we average the propagator at $t$ and that at $T-t$, for baryons we average the propagator for the particle at $t$ and that of the antiparticle at $T-t$. In this work we only calculate the masses of hadrons composed of degenerate mass quarks. 


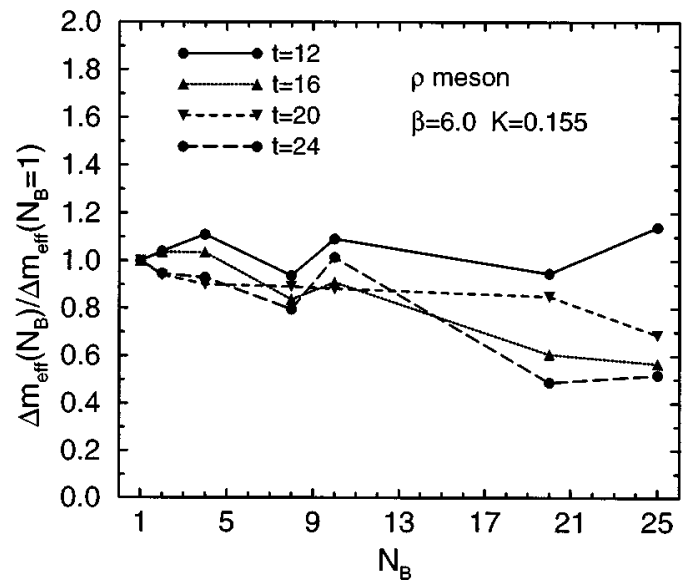

FIG. 1. Statistical errors in effective masses for the $\rho$ meson at $\beta=6.0, K=0.155$ versus the bin size $N_{B}$. The errors are normalized by those for $N_{B}=1$.

The statistical independence of hadron propagators is investigated by the following two methods. (1) We divide the total propagators into bins of $N_{B}$ successive ones and apply the single elimination jackknife method to $N_{\text {conf }} / N_{B}$ blockaveraged propagators. We find that the errors in various quantities do not change significantly even if we change the bin size. Figure 1 shows typical results for the bin size dependence of the error in effective masses. (2) If configurations are independent, we expect that the error obtained for the set of $N$ configurations, $\Delta(N)$, behaves as

$$
\Delta(N) \sim 1 / \sqrt{N}
$$

We check that this behavior is approximately satisfied using the propagators calculated on the first $N$ configurations. Figure 2 shows typical results for the $N$ dependence of the error in effective masses.

\section{HADRON MASSES}

\section{A. Fitting procedure}

The ground state masses of hadrons are extracted by fitting the hadron propagators $G(t)$ to their asymptotic forms:

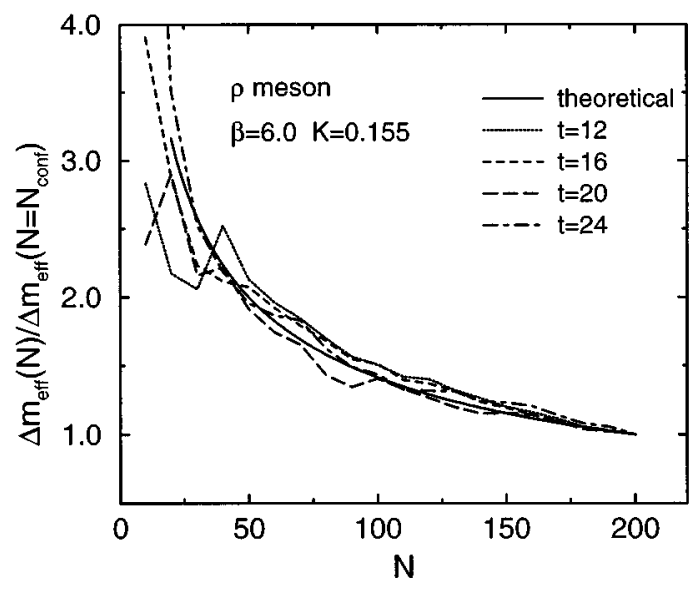

FIG. 2. Statistical errors in effective masses for the $\rho$ meson at $\beta=6.0, K=0.155$ versus the number of configurations $N$. The errors are normalized by those for $N=N_{\text {conf }}=200$.

$$
G_{0}(t)=A\{\exp (-m t)+\exp [-m(T-t)]\}
$$

for mesons and

$$
G_{0}(t)=A \exp (-m t)
$$

for baryons. (We will discuss the masses of excited states later.) We perform least mean square fits taking account of time correlations, minimizing $\chi^{2}$ defined by

$$
\chi^{2}=\sum_{t, t^{\prime}=t_{\min }}^{t_{\max }}\left\{G(t)-G_{0}(t)\right\} C^{-1}\left(t, t^{\prime}\right)\left\{G\left(t^{\prime}\right)-G_{0}\left(t^{\prime}\right)\right\}
$$

where $C^{-1}\left(t, t^{\prime}\right)$ is the inverse of the correlation matrix $C\left(t, t^{\prime}\right) \quad\left(t_{\min } \leqslant t, t^{\prime} \leqslant t_{\max }\right)$. Errors are estimated by two methods. One is the single elimination jackknife method taking account of the correlations among the propagators at different time separations. Another estimate of the error is obtained from the least mean square fit itself. A linear approximation to the fitting function around the minimum of $\chi^{2}$ gives a linear relation between the variance of the fit parameters and the variance of the propagator $G(t)$ for the fitting range $t=t_{\min }-t_{\max }$. The relation leads to the error propagation rule which relates the correlation matrix $C\left(t, t^{\prime}\right)$ to the error (and the correlation) of the fit parameters. We find that the errors obtained by the two methods are of the same order and that the error obtained by the jackknife method is slightly ( $0 \%$ to at most $40 \%)$ larger than that by the least mean square fit. Hereafter we quote the former error for the sake of safety, unless otherwise stated.

\section{B. Fitting ranges and systematic error analyses}

In order to obtain a ground state mass, we have to choose carefully the fitting range $t_{\min }-t_{\max }$ in such a way that the contamination from excited states is negligibly small. We fix $t_{\max }=T / 2$ in order to take into account the data at as large distances as possible. For the purpose of fixing $t_{\min }$, we make fits to a range $t_{0}-T / 2$, varying $t_{0}$ which is a candidate for $t_{\min }$. Then we investigate the $t_{0}$ dependence of the fitted mass $m_{\text {fit }}$ and $\chi^{2} / N_{\mathrm{DF}}, N_{\mathrm{DF}}$ being the number of degrees of freedom, together with the $t$ dependence of the effective mass $m_{\text {eff }}$ defined by

$$
G(t) / G(t+1)=G_{0}\left(t, m_{\mathrm{eff}}(t)\right) / G_{0}\left(t+1, m_{\mathrm{eff}}(t)\right) .
$$

We plot in Figs. 3, 4, and 5, as examples, the results for $\chi^{2} / N_{\mathrm{DF}}, m_{\mathrm{fit}}$, and $m_{\mathrm{eff}}$ at $\beta=6.0, K=0.155$ for the pion, the $\rho$ meson, and the nucleon, respectively. Common features of the time slice dependences of $\chi^{2} / N_{\mathrm{DF}}, m_{\mathrm{fit}}$, and $m_{\mathrm{eff}}$ for all cases including the other cases which are not shown here can be summarized as follows. (Discussion on each particle together with a complete set of figures for effective masses will be given below.)

(1) When we increase $t_{0}$ starting from a small value such as $t_{0}=4, \chi^{2} / N_{\mathrm{DF}}$ decreases rapidly from a large value down to a value around 2.0-0.5 and stabilizes. We denote $t_{0}$ where the stabilization starts as $t_{\chi^{2}}$. The stabilized value of $\chi^{2} / N_{\text {DF }}$ depends on the particle, $\beta$, and $K$. In Table II we give $t_{\chi^{2}}$ and $\chi^{2} / N_{\mathrm{DF}}$ at $t_{\chi^{2}}$. We note that $t_{\chi^{2}}$ values for lighter quarks are smaller than those for heavier quarks. 


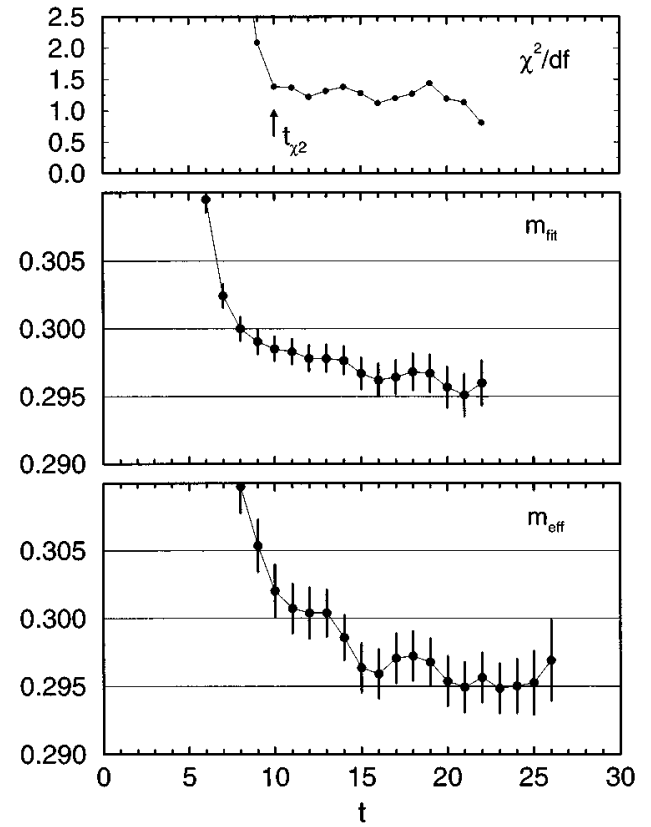

FIG. 3. Fitted mass $m_{\text {fit }}$ for the pion at $\beta=6.0, K=0.155$, obtained from one-mass fit to a range $t-T / 2$ and the value of $\chi^{2} / N_{\mathrm{DF}}$ of the fit versus $t$. The error bars for $m_{\mathrm{fit}}$ are statistical uncertainties estimated by the least mean square fit. Effective masses $m_{\text {eff }}$ with errors estimated by the jackknife method are also given.

From the point of view of the least mean square fit, $t_{\chi^{2}}$ as well as any value of $t>t_{\chi^{2}}$ are candidates for $t_{\min }$.

(2) Although $m_{\text {eff }}(t)$ and $m_{\text {fit }}(t)$ almost stabilize around $t \sim t_{\chi^{2}}$, a clear long plateau in $m_{\text {eff }}$ is rarely seen and the data of $m_{\text {eff }}$ frequently show large and slowly varying fluctuations at large time separations, as shown in the figures. If the fitting range is fixed case by case based on a short plateau of $m_{\text {eff }}$, this may lead to a sizable underestimate of statistical errors.

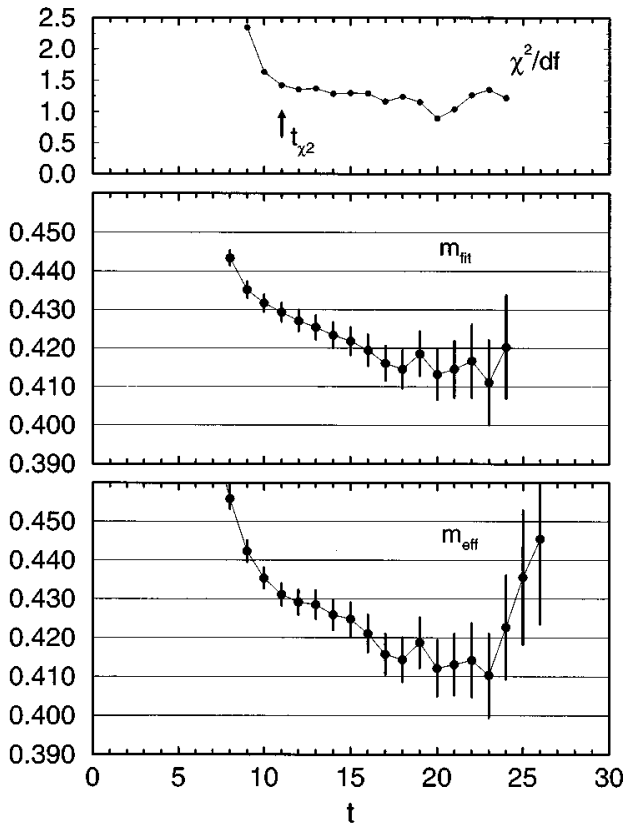

FIG. 4. The same as Fig. 3 for the $\rho$ meson.

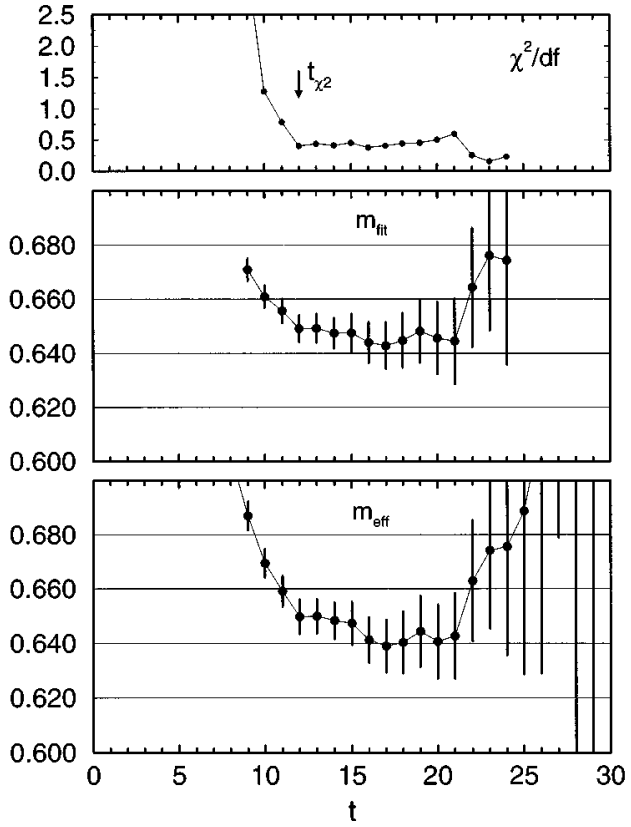

FIG. 5. The same as Fig. 3 for the nucleon.

(3) The value of $m_{\text {eff }}$ in many cases is still decreasing at $t \sim t_{\chi^{2}}$. Similar phenomena are reported by the UKQCD Collaboration [12]. Although probably the large statistical fluctuation mentioned above is a partial cause of this phenomenon, the possibility that excited states still contribute at $t \sim t_{\chi^{2}}$ cannot be excluded. It is difficult to clearly separate out the effects of excited states from the statistical fluctuations.

From these considerations, we do not simply take $t_{\chi^{2}}$ as $t_{\text {min }}$. In order to remove the contamination from excited states as much as possible, we proceed in the following way. We take $t_{\text {min }}$ common to all $K$ 's for the mesons and for the baryons, respectively, at each $\beta$, in order to avoid a subjective choice case by case. Therefore, we require $t_{\min } \geqslant t_{\chi^{2}}$ for all $K$ 's. We further require that $t_{\text {min }}$ always lies in a plateau when a clear plateau is seen in the effective mass plot. In cases where two plateaus are seen (e.g., see Figs. 3-5), we require that $t_{\min }$ is larger than the beginning point of the first plateau. We also pay attention to the consistency between the choices of $t_{\min }$ at two $\beta$ 's in such a way that the ratio of the values of $t_{\min }$ is approximately equal to that of the lattice spacings at the two $\beta$ 's. Thus we have chosen $t_{\min }=12$ (15) for mesons and $t_{\min }=13$ (16) for baryons at $\beta=5.85$ (6.0), respectively. The ratio of $t_{\min }$ at $\beta=5.85$ to that at $\beta=6.0$ is approximately equal to the ratio of the lattice spacings, $a(\beta=5.85) / a(\beta=6.0) \sim 1.2$.

In addition to statistical errors, we estimate the systematic error coming from uncertainties in the choice of fitting range $[5,12]$. Varying $t_{0}$ from $t_{\chi^{2}}$ up to $t_{\text {min }}+4$, we estimate the upper (lower) bound for the systematic error by the difference between the maximum (minimum) value and the central value obtained from the fit with $t_{0}=t_{\min }$. We take $t_{0}$ only up to $t_{\text {min }}+4$, because, when $t_{0}$ is larger than this value, data in the fitting range become too noisy. (For the $\Delta$ baryon at $\beta=6.0$, we vary $t_{0}$ up to $t_{\text {min }}+3$ because a fit with $t_{0}=t_{\text {min }}+4=20$ does not converge.)

In this way we estimate the errors in ground state masses 
TABLE II. $t_{\chi^{2}}$ and $\chi^{2} / N_{\mathrm{DF}}$ at $t_{\chi^{2}}$. See the text for details.

\begin{tabular}{|c|c|c|c|c|c|c|c|c|}
\hline \multicolumn{9}{|c|}{$\beta=5.85$} \\
\hline \multirow[t]{2}{*}{$K$} & \multicolumn{2}{|c|}{$\pi$} & \multicolumn{2}{|c|}{$\rho$} & \multicolumn{2}{|c|}{$N$} & \multicolumn{2}{|c|}{$\Delta$} \\
\hline & $t_{\chi^{2}}$ & $\chi^{2} / N_{\mathrm{DF}}$ & $t_{\chi^{2}}$ & $\chi^{2} / N_{\mathrm{DF}}$ & $t_{\chi^{2}}$ & $\chi^{2} / N_{\mathrm{DF}}$ & $t_{\chi^{2}}$ & $\chi^{2} / N_{\mathrm{DF}}$ \\
\hline 0.1440 & 12 & 0.98 & 12 & 1.32 & 11 & 1.33 & 11 & 1.49 \\
\hline 0.1540 & 10 & 0.90 & 12 & 1.01 & 11 & 1.61 & 11 & 1.61 \\
\hline 0.1585 & 8 & 0.72 & 8 & 2.04 & 9 & 1.36 & 11 & 1.18 \\
\hline 0.1595 & 8 & 0.45 & 8 & 1.73 & 9 & 1.13 & 11 & 1.07 \\
\hline 0.1605 & 8 & 0.46 & 8 & 1.20 & 7 & 1.56 & 9 & 1.30 \\
\hline \multicolumn{9}{|c|}{$\beta=6.0$} \\
\hline \multirow[t]{2}{*}{$K$} & \multicolumn{2}{|c|}{$\pi$} & \multicolumn{2}{|c|}{$\rho$} & \multicolumn{2}{|c|}{$N$} & \multicolumn{2}{|c|}{$\Delta$} \\
\hline & $t_{\chi^{2}}$ & $\chi^{2} / N_{\mathrm{DF}}$ & ${ }^{t} \chi^{2}$ & $\chi^{2} / N_{\mathrm{DF}}$ & ${ }^{t} \chi^{2}$ & $\chi^{2} / N_{\mathrm{DF}}$ & $t_{\chi^{2}}$ & $\chi^{2} / N_{\mathrm{DF}}$ \\
\hline 0.1450 & 15 & 0.55 & 15 & 1.02 & 15 & 0.36 & 15 & 0.57 \\
\hline 0.1520 & 12 & 1.26 & 13 & 0.71 & 15 & 0.38 & 15 & 0.56 \\
\hline 0.1550 & 10 & 1.39 & 11 & 1.42 & 12 & 0.41 & 12 & 0.94 \\
\hline 0.1555 & 10 & 1.35 & 10 & 1.32 & 12 & 0.64 & 12 & 1.22 \\
\hline 0.1563 & 9 & 1.54 & 9 & 0.95 & 10 & 1.21 & 11 & 1.11 \\
\hline
\end{tabular}

due to statistical fluctuations as well as those due to the possibly remaining contamination from excited states which cannot be properly taken into account by the standard least mean square fit with a fixed fitting range. Note that the data are consistent with the implicit assumption that the ground state dominates for $t \geqslant t_{\text {min }}$ when we take into account these systematic errors. Consistency of this assumption is also checked by a two-mass fit discussed in Sec. IV.

\section{Pion masses}

We show $m_{\text {eff }}$ at $\beta=5.85$ and $\beta=6.0$ in Fig. 6 . The pion effective mass has structure with the scale of the standard deviation even for $t \geqslant t_{\chi^{2}}$ : In some cases $m_{\text {eff }}(t)$ exhibits a two-plateau structure or slow monotonic decrease. However, the magnitude of the fluctuation for the pion is much smaller than in the other cases. The resulting systematic error is comparable to the statistical uncertainty. The results of the fits are given in Table III.

\section{D. $\rho$ meson masses}

Fitting to the $\rho$ meson propagator is more problematic than to the pion propagator. Because of this, we will discuss it at some length and compare the results with previous works.

The $\rho$ meson effective mass at $\beta=5.85$ shown in Fig. 7(a) exhibits a plateau for $t \geqslant t_{\chi^{2}}=12$ for the smallest two $K$ 's, while it exhibits peculiar behavior at large $t$ for the largest three $K$ 's: $m_{\mathrm{eff}}(t)$ for $t=17-20$ is larger than that for $t=12-16$ and it drops abruptly at $t=21$. We regard this behavior as due to statistical fluctuations. We find that fits to a range $t=12-t_{\max }$ are stable for $t_{\max }=14-27$. Therefore we choose $t_{\max }=T / 2$ even for these cases. The results of the fits are summarized in Table IV. The systematic error upper bound is 1-2 times larger than the statistical error for the largest three $K$ 's.

Figure 7(b) shows the effective mass at $\beta=6.0$. Except for the smallest $K, m_{\mathrm{eff}}(t)$ is decreasing at $t \sim t_{\chi^{2}}$. The rate of the decrease becomes slow at $t \sim 12$, to exhibit a plateau for two or three time slices. The value of $m_{\text {eff }}$ decreases further up to $t \sim 17$, to attain another plateau. The plateaus are not long enough to determine unambiguously the time slice where the contribution of excited states can be ignored. It should be emphasized again that $\chi^{2} / N_{\mathrm{DF}}$ are almost identical for the fits with both $t_{\min }=12$ and $t_{\min }=17: 1.35$ and 1.16 for $K=0.1550,1.20$ and 1.13 for $K=0.1555$, and 0.77 and 0.76 for $K=0.1563$, respectively. See also Fig. 4. Therefore the value of $\chi^{2}$ does not give a guide to determine $t_{\min }$. The point $t_{\min }=15$ is located between the two pseudo plateaus at $t \sim 12$ and $t \sim 17$. In Table IV are summarized the results for the fits with $t_{\min }=15$ together with the systematic error. Reflecting the slow monotonic decrease of effective masses, the ratio of the systematic error to the statistical error is relatively large: the systematic error amounts to about twice the statistical error for the largest three $K$ 's.

We notice a very intriguing fact: that $m_{\text {fit }}$ by the correlated fits to a range from $t=t_{0}$ to $T / 2$ has a strong correlation with $m_{\text {eff }}$ at $t=t_{0}$. A typical example is seen in Fig. 4. This holds for the other particles also. This means that the result of the fit to a range $t_{0}-T / 2$ is mainly determined from data at $t \sim t_{0}$.

In our previous work [4], we analyzed the same set of $\rho$ meson propagators with uncorrelated fits. Paying attention to the monotonic decrease of effective masses, we made two different fits to estimate the systematic error coming from uncertainties in the choice of fitting range. One is a fit to a range $t \sim 9-11$ at $\beta=5.85(t \sim 12-15$ at $\beta=6.0)$. We called the fit the "preplateau fit." Another is a fit to a range $t \sim 11-t_{\max }$ at $\beta=5.85\left(t \sim 15-t_{\max }\right.$ at $\left.\beta=6.0\right)$, which we called the "plateau fit." The latter fitting ranges correspond approximately to those we adopt in this work. Because $m_{\text {eff }}$ is decreasing, the $\rho$ masses obtained from the correlated fits are systematically larger than those from the uncorrelated fits, due to the fact give in the preceding paragraph. The mass value obtained in this work is between that from the uncorrelated plateau fit and that from the preplateau fit. 

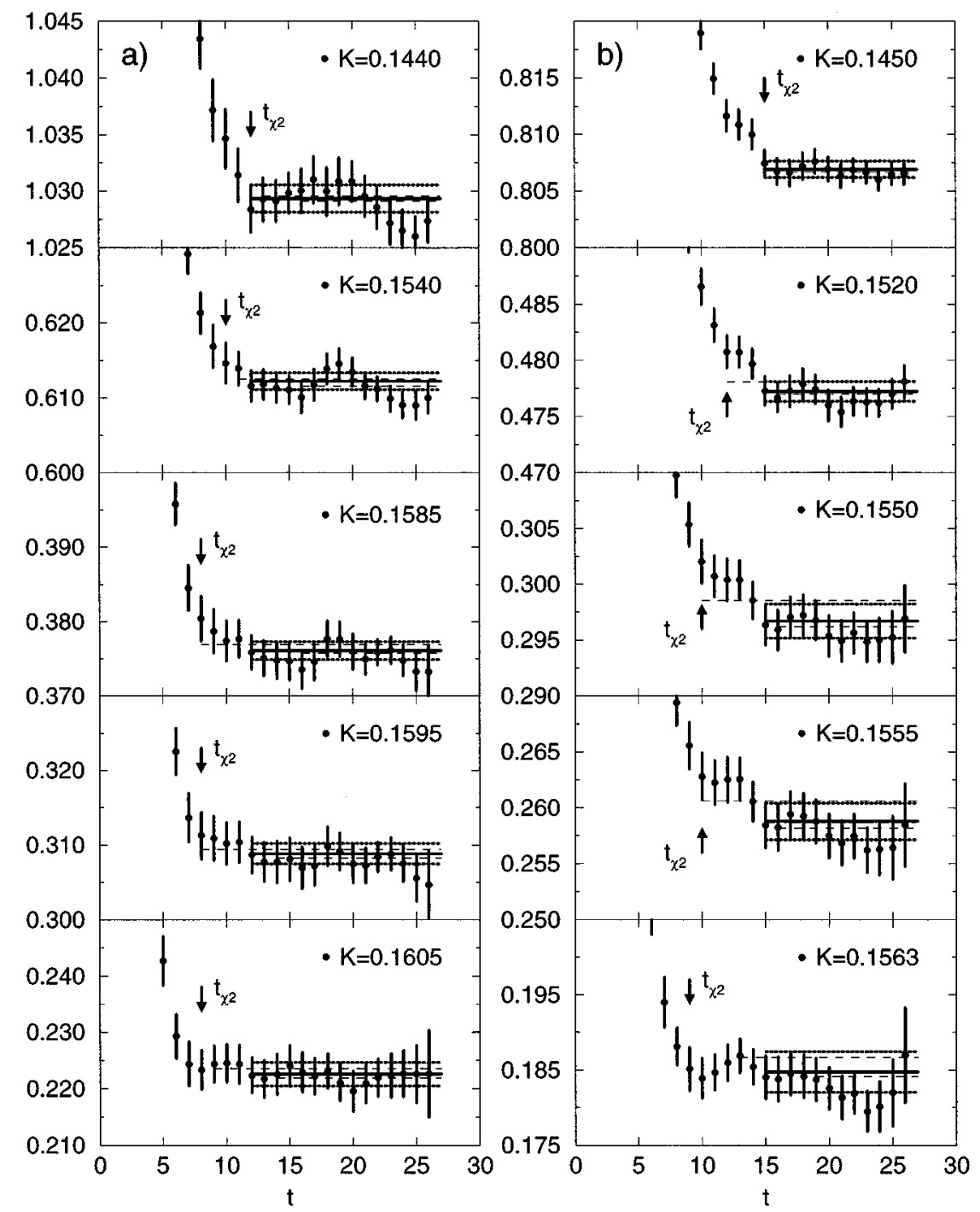

FIG. 6. Effective masses for the pion: (a) $\beta=5.85$ and (b) $\beta=6.0$. The result of the onemass fit is reproduced by the solid line, dotted lines, and dashed lines for the fitted mass, its statistical error, and systematic upper and lower bounds, respectively.

In Table $\mathrm{V}$ we reproduce the results for the $\rho$ meson masses at $\beta=6.0$ for $K=0.155$ and 0.1563 together with those by the APE Collaboration [6,7] and the LANL group [11]. In 1991, the APE Collaboration reported the result obtained on a $24^{3} \times 32$ lattice with a multiorigin $7^{3}$ cubic source [7]. Then we made simulations for the same spatial size with larger temporal extent [4], $24^{3} \times 54$, using the point source. For $K=0.155$, the values of $m_{\mathrm{eff}}$ at $t \sim 10$ are in close agreement with APE's. Consequently the result 0.4280(33) obtained from the preplateau fit $(t=12-15)$ agreed with the

TABLE III. Pion masses in lattice units. In parentheses are errors estimated by the jackknife method. Errors given in the form ${ }_{-}^{+}$- upper are for the fitting range dependent upper and lower bounds.

\begin{tabular}{ccrccr}
\hline \hline \multicolumn{3}{c}{$\beta=5.85$} & & \multicolumn{3}{c}{$\beta=6.0$} \\
$K$ & $m_{\pi}$ & $\chi^{2} / N_{\mathrm{DF}}$ & $K$ & $m_{\pi}$ & $\chi^{2} / N_{\mathrm{DF}}$ \\
\hline 0.1440 & $1.0293(12)_{-2}^{+2}$ & $13.7 / 14$ & 0.1450 & $0.8069(7)_{-2}^{+0}$ & $6.1 / 11$ \\
0.1540 & $0.6122(11)_{-6}^{+3}$ & $10.9 / 14$ & 0.1520 & $0.4772(9)_{-2}^{+9}$ & $11.5 / 11$ \\
0.1585 & $0.3761(12)_{-4}^{+8}$ & $7.4 / 14$ & 0.1550 & $0.2967(15)_{-5}^{+18}$ & $14.1 / 11$ \\
0.1595 & $0.3088(14)_{-6}^{+6}$ & $5.8 / 14$ & 0.1555 & $0.2588(16)_{-6}^{+18}$ & $17.0 / 11$ \\
0.1605 & $0.2226(21)_{-7}^{+10}$ & $6.0 / 14$ & 0.1563 & $0.1847(27)_{-6}^{+20}$ & $20.9 / 11$ \\
\hline \hline
\end{tabular}

APE result 0.429 (3) within one standard deviation. However, the result $0.4169(48)$ from the plateau fit $(t=15-27)$ was smaller by approximately twice the statistical error. We regarded the latter as more reliable. At that time there was a report that the mass value appears to depend on the type of source adopted [13]. Therefore, in order to clarify whether the origin of the discrepancy between our result and the APE result is due to the different type of source, we made calculations at $K=0.155$ for 400 configurations [5] using the point source, the wall source, and the source adopted by the APE Collaboration. The results obtained from correlated fits for the three different sources agreed with each other: 0.4201(29), 0.4228(19), and 0.4249(19) for the point source, the wall source, and the multiorigin source, respectively. The recent result reported by the LANL group, 0.422(3) [11], is consistent with these numbers. It is probable that the slightly larger value by the APE Collaboration is due to the small temporal extent. The APE Collaboration has also made simulations using both the point source and the multicube source [6] with larger temporal size and smaller spatial size: $18^{3} \times 64$. Their results $0.430(10)$ and $0.428(8)$ are consistent with other results within relatively large errors, although the central values are slightly higher than the results by other 

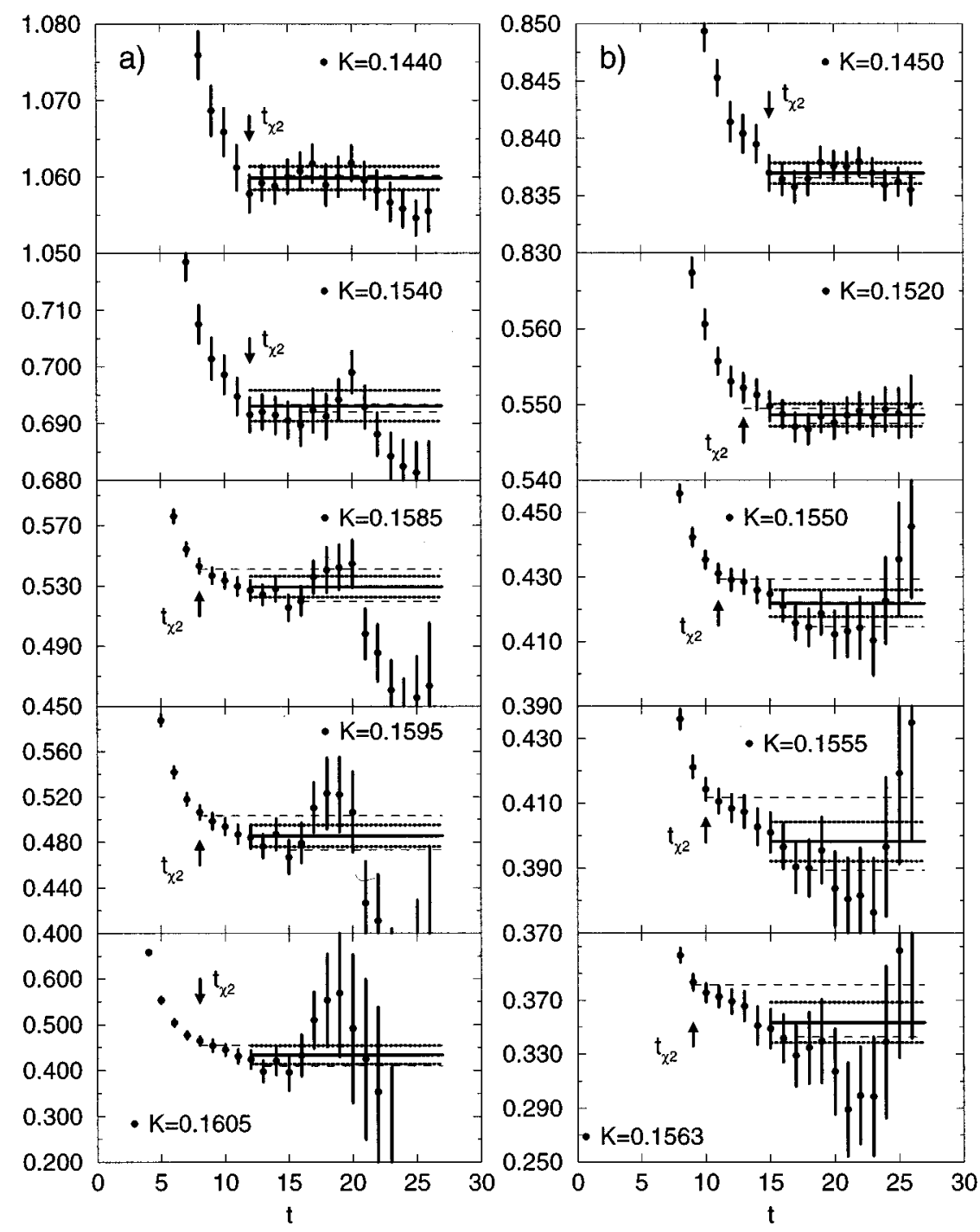

FIG. 7. The same as Fig. 6 for the $\rho$ meson: (a) $\beta=5.85$ and (b) $\beta=6.0$. groups. The slightly larger central values may be due to the small spatial size. For $K=0.1563$, the results obtained from the correlated fit in this work are consistent with those by the APE Collaboration and the LANL group, albeit with large errors in the results.

\section{E. Baryon masses}

Figure 8 shows effective masses for the nucleon at $\beta=5.85$ and $\beta=6.0$. Decrease of $m_{\text {eff }}$ at $t \sim t_{\chi^{2}}$ is not conspicuous compared with the case of the $\rho$ meson. However, we see a two-plateau structure for the cases of $K=0.1585$

TABLE IV. The same as Table III for the $\rho$ meson.

\begin{tabular}{cccccr}
\hline \hline \multicolumn{3}{c}{$\beta=5.85$} & & \multicolumn{3}{c}{$\beta=6.0$} \\
$K$ & $m_{\rho}$ & $\chi^{2} / N_{\mathrm{DF}}$ & $K$ & $m_{\rho}$ & $\chi^{2} / N_{\mathrm{DF}}$ \\
\hline 0.1440 & $1.0598(15)_{-1}^{+4}$ & $18.5 / 14$ & 0.1450 & $0.8370(9)_{-4}^{+2}$ & $11.2 / 11$ \\
0.1540 & $0.6931(27)_{-11}^{+3}$ & $14.2 / 14$ & 0.1520 & $0.5486(15)_{-11}^{+9}$ & $6.6 / 11$ \\
0.1585 & $0.5294(69)_{-1150}^{+115}$ & $28.9 / 14$ & 0.1550 & $0.4218(42)_{-73}^{+75}$ & $14.3 / 11$ \\
0.1595 & $0.4856(96)_{-123}^{+176}$ & $23.2 / 14$ & 0.1555 & $0.3982(61)_{-90}^{+135}$ & $12.4 / 11$ \\
0.1605 & $0.434(20)_{-24}^{+21}$ & $14.6 / 14$ & 0.1563 & $0.353(15)_{-11}^{+28}$ & $7.6 / 11$ \\
\hline \hline
\end{tabular}

and 0.1595 at $\beta=5.85$ and $K=0.155$ (see also Fig. 5) and 0.1555 at $\beta=6.0$. The choice $t_{\min }=13$ (16) for $\beta=5.85$ (6.0) corresponds to selecting the first (last) plateau as correct for the case where two plateaus are observed. Table VI summarizes the results of the fits.

For $\Delta$, a monotonic decrease of effective masses at $t \sim t_{\chi^{2}}$ or a two-plateau structure is seen for $K=0.1595$ and 0.1605 at $\beta=5.85$ and for $K=0.1550$ and 0.1563 at $\beta=6.0$. Effective mass plots are shown in Fig. 9. The results of the fits are summarized in Table VII.

In Table $\mathrm{V}$, the baryon masses at $\beta=6.0$ for $K=0.155$ and 0.1563 together with those by the APE Collaboration and the LANL group are reproduced. The nucleon masses reported by the three groups agree within the statistical uncertainties. The $\Delta$ masses for $K=0.155$ are slightly scattered: Our result is higher than the LANL result by two standard deviations. However, note that the values of the $\Delta$ mass obtained on 400 configurations [5] [0.7054(95), 0.7008(57), and $0.7128(191)$ for the point source, the wall source, and the multiorigin source, respectively] are in good agreement with the LANL result. Therefore we think that the difference between the LANL result and our present result is due to statistical errors. 
TABLE V. Comparison of hadron masses in lattice units at $\beta=6.0, K=0.155$ and 0.1563 .

\begin{tabular}{|c|c|c|c|c|}
\hline \multicolumn{5}{|c|}{$K=0.155$} \\
\hline & $\pi$ & $\rho$ & $N$ & $\Delta$ \\
\hline This work $24^{3} \times 54$ & $0.2967(15)$ & $0.4218(42)$ & $0.6440(85)$ & $0.728(11)$ \\
\hline APE $24^{3} \times 32[7]$ & $0.298(2)$ & $0.429(3)$ & $0.647(6)$ & $0.745(15)$ \\
\hline APE $18^{3} \times 64[6]$ smear & $0.297(2)$ & $0.430(10)$ & & \\
\hline local & $0.297(2)$ & $0.428(8)$ & & \\
\hline LANL $32^{3} \times 64[11]$ & $0.297(1)$ & $0.422(3)$ & $0.641(4)$ & $0.706(8)$ \\
\hline \multicolumn{5}{|c|}{$K=0.1563$} \\
\hline & $\pi$ & $\rho$ & $N$ & $\Delta$ \\
\hline This work $24^{3} \times 54$ & $0.1847(27)$ & $0.353(15)$ & $0.536(30)$ & $0.670(53)$ \\
\hline APE $24^{3} \times 32[7]$ & $0.184(3)$ & $0.377(8)$ & $0.522(14)$ & $0.636(45)$ \\
\hline LANL $32^{3} \times 64[11]$ & $0.185(1)$ & $0.363(9)$ & $0.540(12)$ & $0.631(27)$ \\
\hline
\end{tabular}

\section{F. Finite lattice effects}

The linear extension of the lattice in the spatial directions is $2.45(2.03) \mathrm{fm}$ at $\beta=5.85(6.0)$, when we use $a^{-1}=1.93$ (2.33) GeV determined from $m_{\rho}$ (see Sec. V). These values are much larger than twice the electromagnetic radius of the nucleon, $2 \times 0.82 \mathrm{fm}$. We also note that our results on the lattice with spatial volume $24^{3}$ agree well with those on a lattice with $32^{3}$ [11], as discussed above. Therefore we do not take into account in this work finite lattice effects, which are supposed to be small.

\section{G. Mass ratios}

The mass ratio $m_{N} / m_{\rho}$ is plotted versus $\left(m_{\pi} / m_{\rho}\right)^{2}$ in Fig. 10. The values of the mass ratio are given in Table VIII. The
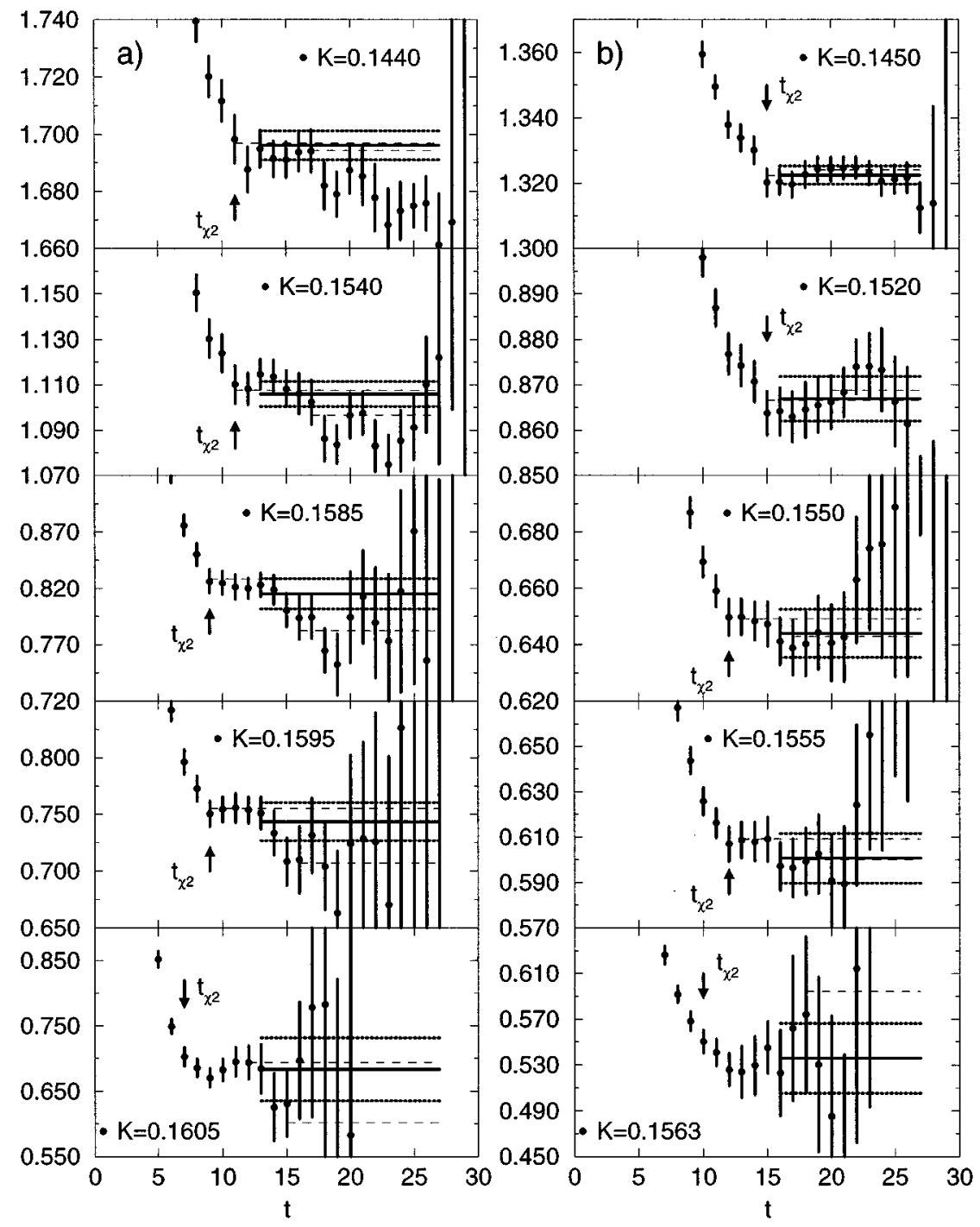

FIG. 8. The same as Fig. 6 for the nucleon: (a) $\beta=5.85$ and (b) $\beta=6.0$. 
TABLE VI. Nucleon masses in lattice units. In parentheses are errors estimated by the jackknife method. Errors given in the form ${ }_{-}^{+}$-lower are for the fitting range dependent upper and lower bounds.

\begin{tabular}{cccccr}
\hline \hline \multicolumn{3}{c}{$\beta=5.85$} & & \multicolumn{3}{c}{$\beta=6.0$} \\
$K$ & $m_{N}$ & $\chi^{2} / N_{\mathrm{DF}}$ & $K$ & $m_{N}$ & $\chi^{2} / N_{\mathrm{DF}}$ \\
\hline 0.1440 & $1.6961(50)_{-18}^{+7}$ & $15.8 / 13$ & 0.1450 & $1.3225(28)_{-2}^{+15}$ & $3.8 / 10$ \\
0.1540 & $1.1060(55)_{-94}^{+15}$ & $22.3 / 13$ & 0.1520 & $0.8669(49)_{-3}^{+19}$ & $4.2 / 10$ \\
0.1585 & $0.815(13)_{-33}^{+13}$ & $17.5 / 13$ & 0.1550 & $0.6440(85)_{-12}^{+53}$ & $3.8 / 10$ \\
0.1595 & $0.744(17)_{-36}^{+12}$ & $18.1 / 13$ & 0.1555 & $0.6007(109)_{-7}^{+84}$ & $6.2 / 10$ \\
0.1605 & $0.683(48)_{-82}^{+10}$ & $23.4 / 13$ & 0.1563 & $0.536(30)_{-0}^{+58}$ & $15.7 / 10$ \\
& & & & &
\end{tabular}

value of $m_{N} / m_{\rho}$ at $\beta=6.0$ is systematically smaller than that at $\beta=5.85$, although the results at the two $\beta$ 's agree within the statistical uncertainty except for the case of the heaviest quark $\left[\left(m_{\pi} / m_{\rho}\right)^{2} \sim 0.94\right]$.

\section{EXCITED STATE MASSES}

In addition to the masses of the ground states, we study the masses of the first excited states for the $\rho$ meson and the nucleon. To this end, we perform two-mass fits to the corresponding propagators, varying $t_{\min }$. Our results for the $\rho$ meson are shown in Fig. 11 for $\beta=5.85, K=0.1585$, and in
Fig. 12 for $\beta=6.0, K=0.155$. The results for the nucleon are given in Figs. 13 and 14 for $\beta=5.85$ and 6.0, respectively. We find the following:

(1) $\chi^{2} / N_{\mathrm{DF}}$ is stable and small $(\sim 1-2)$ for $t_{\min } \geqslant 4$ (5) in the case of the $\rho$ meson and for $t_{\min } \geqslant 5$ (6) in the case of the nucleon at $\beta=5.85(6.0)$, respectively; (2) when $\chi^{2} / N_{\mathrm{DF}}$ is small, the ground state masses $m_{0}$ from the two-mass fit are consistent with those from the one-mass fit within the errors, although the errors for $m_{0}$ from the two-mass fit become extremely large at large $t_{\mathrm{min}}$; (3) although $\chi^{2} / N_{\mathrm{DF}}$ is stable, the mass of the first excited state, $m_{1}$, is in general quite unstable. For example, for the $\rho$ meson at $\beta=5.85$, the value of $m_{1}$ decreases from 1.5 for $t_{\min }=3$ to 0.6 for $t_{\min }=9$ (cf. Fig. 11). Similar behavior is also seen in the results for the $\rho$ meson at $\beta=6.0$ (Fig. 12) and the nucleon at $\beta=5.85$ (Fig. 13). The case of the nucleon at $\beta=6.0$ is exceptional: $m_{1}$ is relatively stable (Fig. 14).

Under these circumstances, we select two $t_{\min }$ 's which give $m_{0}$ consistent with the result of the one-mass fit, under the condition that the errors are small. We then investigate whether the results for the excited state mass are consistent with the corresponding experimental values.

In Figs. 15 and 16 are shown the first excited state masses

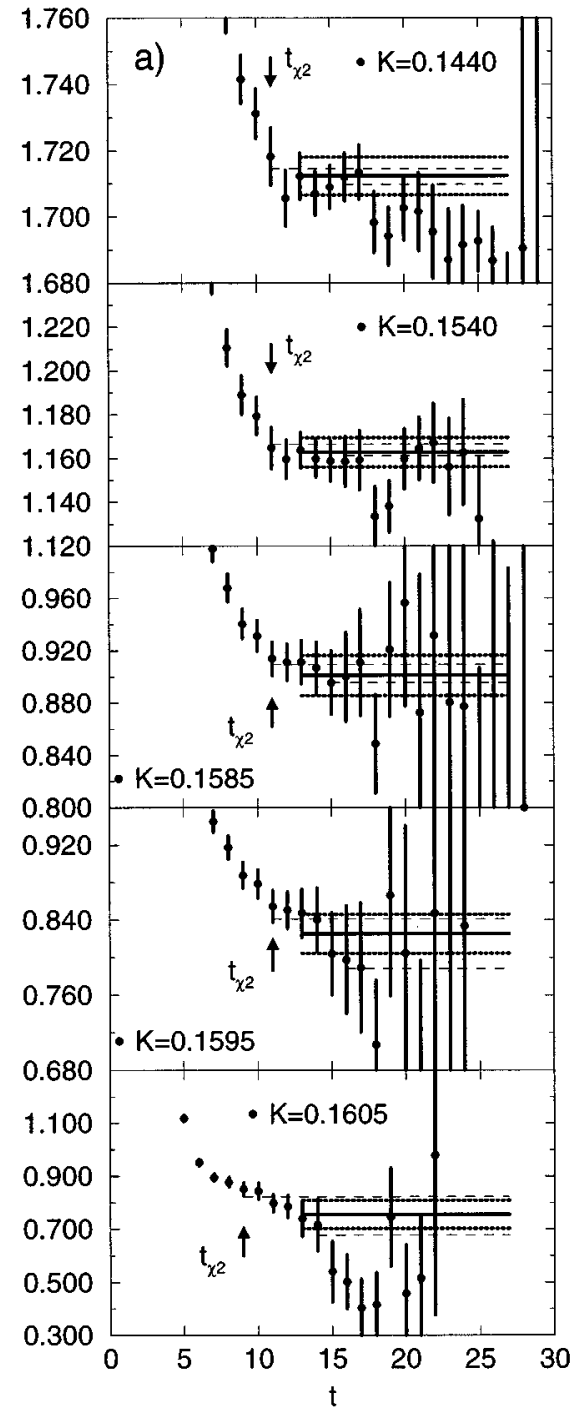

FIG. 9. The same as Fig. 6 for the $\Delta$ baryon: (a) $\beta=5.85$ and (b) $\beta=6.0$. 
TABLE VII. The same as Table VI for the $\Delta$ baryon.

\begin{tabular}{cccccr}
\hline \hline \multicolumn{3}{c}{$\beta=5.85$} & & & $\beta=6.0$ \\
$K$ & $m_{\Delta}$ & $\chi^{2} / N_{\mathrm{DF}}$ & $K$ & $m_{\Delta}$ & $\chi^{2} / N_{\mathrm{DF}}$ \\
\hline 0.1440 & $1.7124(57)_{-25}^{+21}$ & $17.7 / 13$ & 0.1450 & $1.3404(29)_{-1}^{+22}$ & $6.2 / 10$ \\
0.1540 & $1.1629(67)_{-15}^{+37}$ & $20.3 / 13$ & 0.1520 & $0.9112(41)_{-0}^{+51}$ & $6.0 / 10$ \\
0.1585 & $0.9011(153)_{-57}^{+83}$ & $16.5 / 13$ & 0.1550 & $0.7278(109)_{-0}^{+188}$ & $12.1 / 10$ \\
0.1595 & $0.825(21)_{-37}^{+16}$ & $15.1 / 13$ & 0.1555 & $0.7001(159)_{-10}^{+336}$ & $15.3 / 10$ \\
0.1605 & $0.755(53)_{-78}^{+67}$ & $19.4 / 13$ & 0.1563 & $0.670(53)_{-41}^{+61}$ & $9.0 / 10$ \\
\hline \hline
\end{tabular}

of the $\rho$ meson obtained from the fits with $t_{\min }=5$ and 6 (8 and 9) versus $1 / K$ at $\beta=5.85$ (6.0), respectively. (A twomass fit with $t_{\min }=9$ for the largest $K$ at $\beta=6.0$ does not converge. Therefore the corresponding data are missing in the figure.) We give in the figures the experimental values for the masses of $\rho(1450)$ and $\phi(1680)$, which are the first excited states of the vector mesons. The mass of $\phi(1680)$ is plotted at the third largest $K$, because this value of $K$ corresponds to the strange quark mass as mentioned in Sec. V G. Apparently the results for the excited state mass depend strongly on the value of $t_{\min }$. For quarks lighter than the strange quark, the excited state mass obtained with smaller $t_{\text {min }}$ is much larger than experiment, while that with larger $t_{\text {min }}$ is consistent with experiment within large statistical errors. Therefore, although the value of $m_{1}$ is unstable, there exist two-mass fits to the $\rho$ propagators which give both a ground state mass consistent with the one-mass fit and a first excited state mass consistent with experiment.

Figure 17 shows the masses of excited states of the nucleon at $\beta=5.85$ versus $1 / K$. The excited state masses obtained from the fit with $t_{\min }=7$ are much smaller than those with $t_{\min }=6$. (A two-mass fit with $t_{\min }=7$ for the largest $K$ does not converge.) We expect that the mass difference between the ground state and the first excited state depends only weakly on the quark mass, because the mass difference for the spin $1 / 2$ baryon satisfies this property. The mass difference for the nucleon is $m_{N(1440)}-m_{N(940)}=500 \mathrm{MeV}$. The figure shows that the excited state masses with $t_{\min }=7$ lie approximately $500 \mathrm{MeV}$ higher than the ground state masses. Therefore there exist two-mass fits whose results do not contradict with experiment also for the nucleon at $\beta=5.85$.

In Fig. 18 we show the excited state masses of the nucleon at $\beta=6.0$ with $t_{\min }=7$. The masses of the first excited state lie much more than $500 \mathrm{MeV}$ above the ground

TABLE VIII. Mass ratios $m_{\pi} / m_{\rho}$ and $m_{N} / m_{\rho}$. The errors quoted are statistical only and are estimated by the jackknife method.

\begin{tabular}{cllclll}
\hline \hline \multicolumn{3}{c}{$\beta=5.85$} & & \multicolumn{3}{c}{$\beta=6.0$} \\
\multicolumn{1}{c}{$K$} & \multicolumn{1}{c}{$m_{\pi} / m_{\rho}$} & $m_{N} / m_{\rho}$ & \multicolumn{1}{c}{$K$} & \multicolumn{1}{c}{$m_{\pi} / m_{\rho}$} & $m_{N} / m_{\rho}$ \\
\hline 0.1440 & $0.9712(8)$ & $1.6004(45)$ & 0.1450 & $0.9641(5)$ & $1.5801(25)$ \\
0.1540 & $0.8833(32)$ & $1.5956(82)$ & 0.1520 & $0.8699(21)$ & $1.5802(79)$ \\
0.1585 & $0.7104(90)$ & $1.540(29)$ & 0.1550 & $0.7033(69)$ & $1.527(21)$ \\
0.1595 & $0.636(12)$ & $1.531(42)$ & 0.1555 & $0.650(10)$ & $1.509(31)$ \\
0.1605 & $0.513(25)$ & $1.57(12)$ & 0.1563 & $0.523(23)$ & $1.52(10)$ \\
\hline \hline
\end{tabular}

state masses. As mentioned before, two-mass fits for the nucleon at $\beta=6.0$ are stable and therefore the values of the excited state mass do not change much even if we take other $t_{\text {min }}$ values. When we recall that there exists a fit which gives a reasonable excited state mass at $\beta=5.85$, this situation is puzzling. One possible origin for the heavy excited state mass at $\beta=6.0$ is a finite size effect, because the physical volume is smaller at $\beta=6.0$. There remains a possibility that when we simulate on a larger lattice, a two-mass fit with larger $t_{\min }$ will give a value consistent with the nucleon excited state mass.

There are several published data for the mass of excited states $[5,7,8,15,16]$. In Table IX, we reproduce the results for the ratio of the excited state mass to the ground state mass, selecting the quark mass corresponding approximately to the strange quark mass. For the $\rho$ meson, except our results in this work with $t_{\min }=6(9)$ at $\beta=5.85(6.0)$ and the result for the wall source in Ref. [5], the reported ratios are considerably larger than the corresponding experimental value $m_{\phi(1680)} / m_{\phi(1020)}=1.65$. For the nucleon, the mass ratios reported by the APE Collaboration and the UKQCD Collaboration are considerably larger than our result. One possible origin of the differences is the choice of fitting range. Because the two-mass fit is very unstable, we certainly have to employ a more efficient way to extract reliable values for the excited state masses.

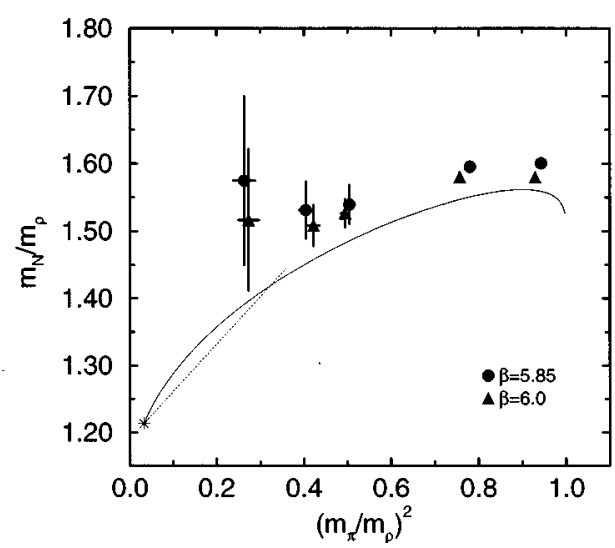

FIG. 10. Nucleon to $\rho$ mass ratio versus pion to $\rho$ mass ratio squared. The errors shown are statistical only. The solid curve is obtained from phenomenological mass formulas [28]. The dotted line is obtained by assuming that $m_{N} / m_{\rho}$ and $\left(m_{\pi} / m_{\rho}\right)^{2}$ are linear in the quark mass. The experimental value is marked with a star. 


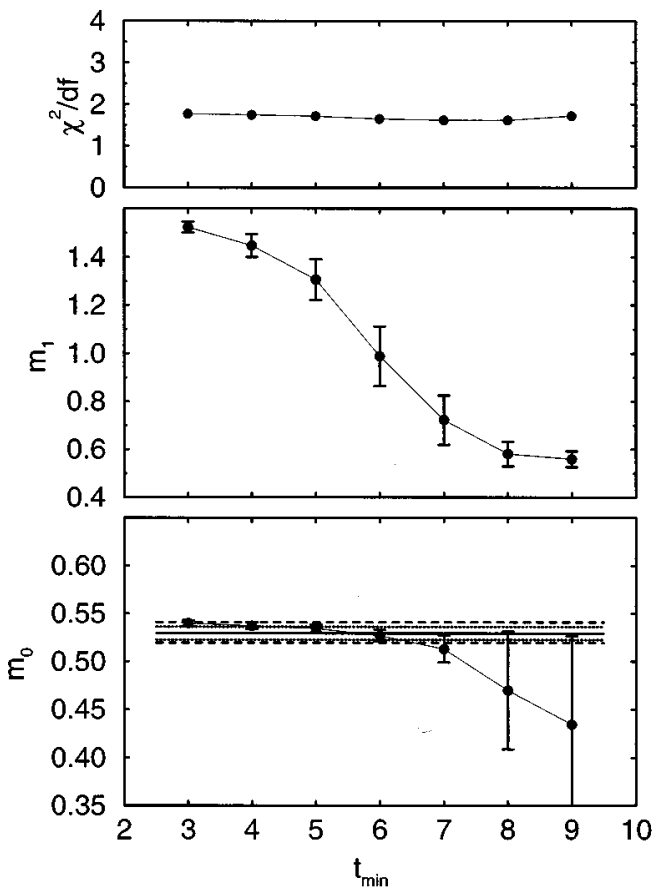

FIG. 11. Masses of the ground state and the excited state for the $\rho$ meson at $\beta=5.85, K=0.1585$ together with the value of $\chi^{2} / N_{\mathrm{DF}}$ of the two-mass fits versus $t_{\min }$. The error bars are statistical uncertainties estimated by the least mean square fit. The result of the one-mass fit is reproduced by the solid line, dotted lines, and dashed lines for the fitted mass, its statistical error, and systematic upper and lower bounds, respectively. Note the difference in the scale of the plots for $m_{0}$ and $m_{1}$.

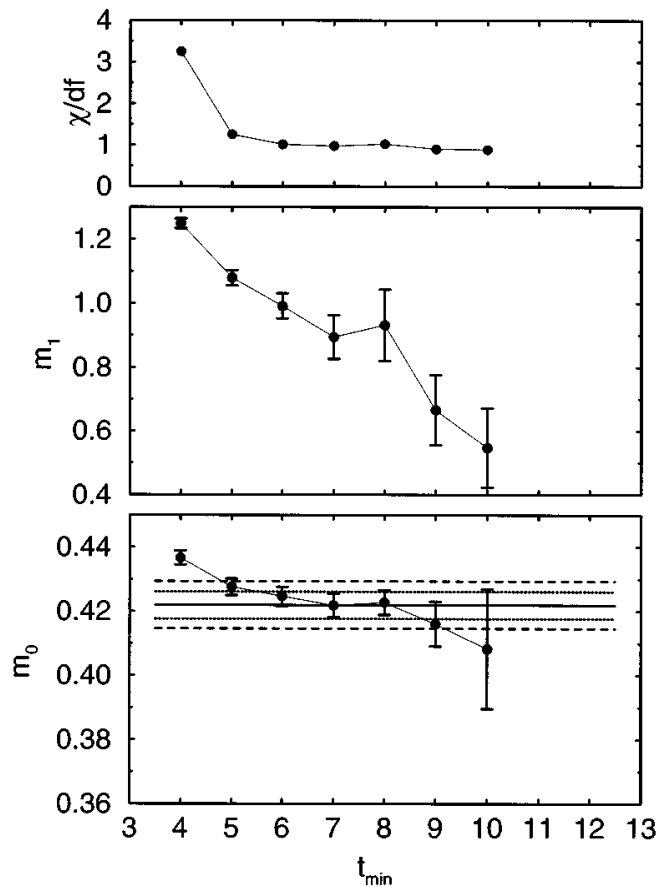

FIG. 12. The same as Fig. 11 for the $\rho$ meson at $\beta=6.0$, $K=0.155$.

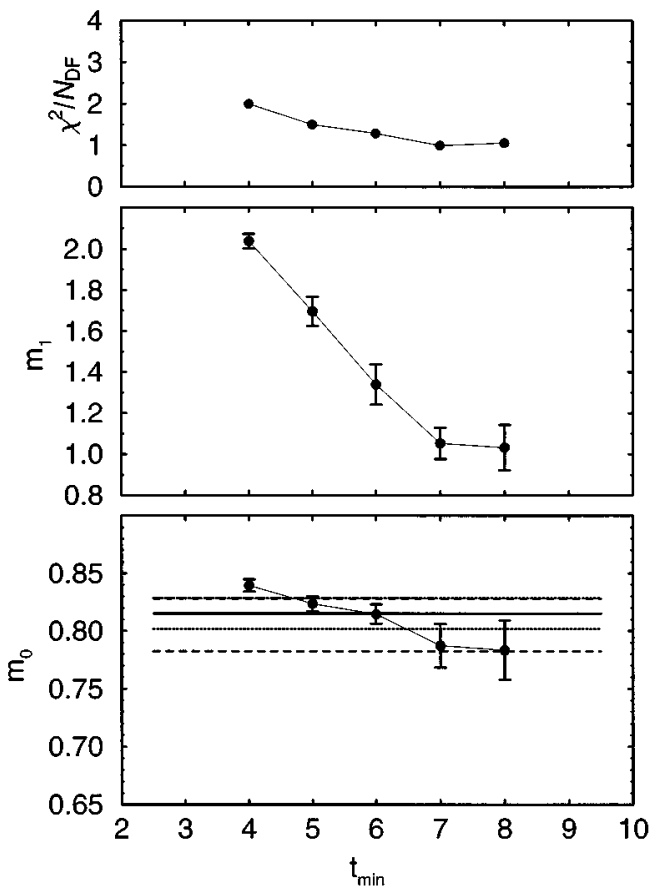

FIG. 13. The same as Fig. 11 for the nucleon at $\beta=5.85$, $K=0.1585$.

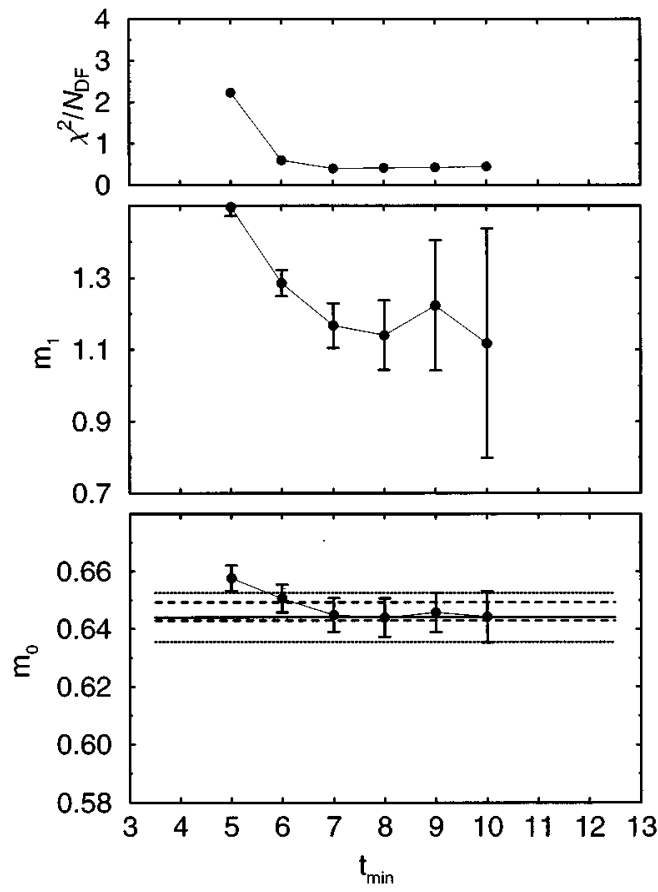

FIG. 14. The same as Fig. 11 for the nucleon at $\beta=6.0$, $K=0.155$. 


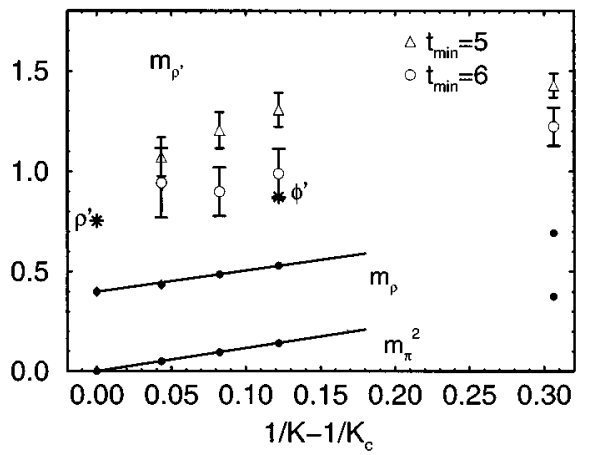

FIG. 15. Mass of the excited state of the $\rho$ meson (denoted by $\left.\rho^{\prime}\right)$ at $\beta=5.85$ versus $1 / K-1 / K_{c}$. The corresponding experimental values are marked with stars. The data for $m_{\rho}$ and $m_{\pi}^{2}$ are taken from the results of one-mass fits. With the scale of the plot, the results for $m_{\rho}$ from two-mass fits are indistinguishable from the one-mass fit results.

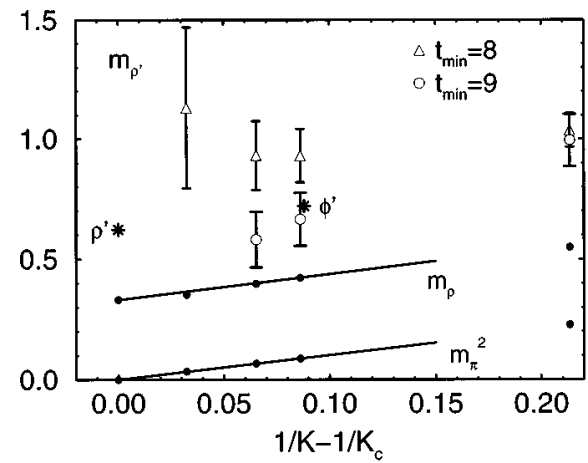

FIG. 16. The same as Fig. 15 for $\beta=6.0$.

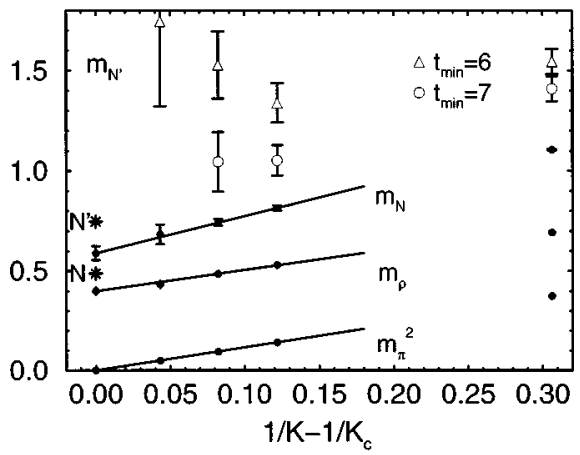

FIG. 17. Mass of excited state of the nucleon (denoted by $N^{\prime}$ ) at $\beta=5.85$ versus $1 / K-1 / K_{c}$. The experimental values for masses of the nucleon $(N)$ and its excited state $\left(N^{\prime}\right)$ are marked with stars. The data for $m_{N}, m_{\rho}$, and $m_{\pi}^{2}$ are taken from the results of onemass fits. With the scale of the plot, the results for $m_{N}$ from twomass fits are indistinguishable from the one-mass fit results.

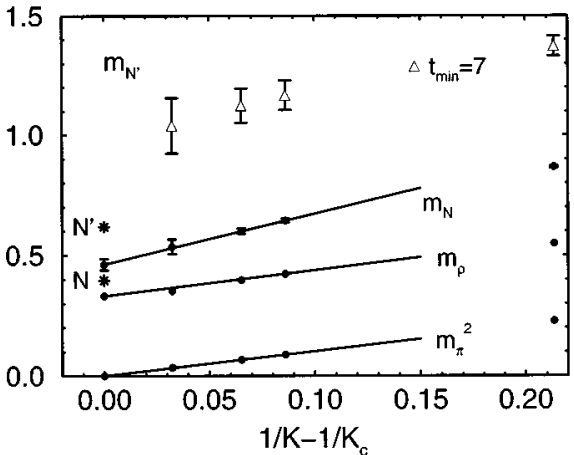

FIG. 18. The same as Fig. 17 for $\beta=6.0$.

\section{MASSES OF HADRONS WITH PHYSICAL LIGHT QUARKS}

\section{A. Extrapolation procedure}

Extrapolation of hadron masses to the chiral limit is done taking into account the correlation among the masses at different values of hopping parameter. First we consider a least mean square fit to minimize

$$
\begin{aligned}
\chi^{2}= & \sum_{t, t^{\prime}, K, K^{\prime}}\left\{G(t, K)-G_{0}(t, K)\right\} C^{-1}\left(t, K ; t^{\prime}, K^{\prime}\right)\left\{G\left(t^{\prime}, K^{\prime}\right)\right. \\
& \left.-G_{0}\left(t^{\prime}, K^{\prime}\right)\right\}
\end{aligned}
$$

where $G_{0}(t, K)=A(K) e^{-m(K) t}$ is the fitting function to the hadron propagator $G(t, K)$ and $C^{-1}$ is the inverse of the full correlation matrix $C\left(t, K ; t^{\prime} ; K^{\prime}\right)$. A linear approximation to the fitting function around the minimum of $\chi^{2}$ gives the relation between the error matrix $\Sigma$ for the fit parameters and the correlation matrix $C\left(t, K ; t^{\prime}, K^{\prime}\right)$ for propagators:

$$
\Sigma=\left(D^{T} C^{-1} D\right)^{-1},
$$

where $D$ is the Jacobian defined by

$$
D_{t, K ; A\left(K^{\prime}\right), m\left(K^{\prime}\right)}=\left[\partial G_{0}(t, K) / \partial A\left(K^{\prime}\right), \partial G_{0}(t, K) / \partial m\left(K^{\prime}\right)\right] .
$$

( $D$ is diagonal with respect to $K$.) The full least mean square fit to minimize $\chi^{2}$ in Eq. (14) is different from the set of least mean square fits for each $K$ to minimize $\chi^{2}$,s in Eq. (12): The masses and amplitudes obtained by the two methods are in general different. We take those obtained from the fits to each propagator for evaluation of the Jacobian. ${ }^{1}$

For extrapolation, we minimize $\chi^{2}$ given by

$$
\chi^{2}=\sum_{K}\{m(K)-f(K)\} \Sigma^{-1}\left(K, K^{\prime}\right)\left\{m\left(K^{\prime}\right)-f\left(K^{\prime}\right)\right\}
$$

\footnotetext{
${ }^{1}$ We have checked that the error matrix thus obtained is very close to that obtained using the Jacobian at the absolute minimum of Eq. (14). Consequently, the difference in the extrapolated values obtained using two error matrices is at most $5 \%$ of their statistical uncertainties.
} 
TABLE IX. Ratios of the excited state mass to the ground state mass. We have taken the quark mass corresponding approximately to the strange quark mass.

\begin{tabular}{|c|c|c|c|c|c|}
\hline & \multirow[b]{2}{*}{$\beta$} & \multicolumn{2}{|c|}{$\rho$ meson } & \multicolumn{2}{|c|}{ Nucleon } \\
\hline & & Comment & Ratio & Comment & Ratio \\
\hline \multirow[t]{4}{*}{ This work } & 5.85 & $t_{\min }=5$ & $2.47(16)$ & $t_{\min }=6$ & $1.64(12)$ \\
\hline & & $t_{\min }=6$ & $1.87(24)$ & $t_{\min }=7$ & $1.29(10)$ \\
\hline & 6.0 & $t_{\min }=8$ & $2.21(27)$ & $t_{\min }=7$ & $1.81(10)$ \\
\hline & & $t_{\min }=9$ & $1.58(26)$ & & \\
\hline APE [7] & 6.0 & & $2.13(21)$ & & $2.13(4)$ \\
\hline UKQCD [15] & 6.2 & & $2.53(16)$ & & $2.01(16)$ \\
\hline APE [8] & 6.3 & & $1.93(10)$ & & $1.93(12)$ \\
\hline UKQCD [16] & 6.2 Clover & & $2.23(14)$ & & \\
\hline \multirow[t]{2}{*}{ QCDPAX [5] } & 6.0 & Point & $1.99(15)$ & Point & $1.55(20)$ \\
\hline & & Wall & $1.70(26)$ & Wall & $1.47(21)$ \\
\hline Experimental value & & & 1.65 & & \\
\hline
\end{tabular}

where the correlation matrix $\Sigma\left(K, K^{\prime}\right)$ is the submatrix among the masses of the full error matrix $\Sigma$ and $f(K)$ is the fitting function. [For the pion, $m(K)$ is replaced by $m^{2}(K)$ with appropriate replacement of $\Sigma^{-1}\left(K, K^{\prime}\right)$.]

\section{B. Linear extrapolation to the chiral limit}

We fit the data of the mass squared for the pion and the mass for the other hadrons at the largest three $K$ 's to a linear function of $1 / K ; f(K)=a_{0}+a_{1} / K$. We find that the quality of the linear fit is good in the sense that $\chi^{2} / N_{\mathrm{DF}}<2$ $\left(N_{\mathrm{DF}}=1\right.$ in this case) and therefore we do not study in this work the effects of possible chiral logarithms $[17,18]$. We summarize the fit parameters together with $\chi^{2} / N_{\mathrm{DF}}$ in Table $\mathrm{X}$. The linear extrapolations of hadron masses at $\beta=5.85$ and 6.0 are shown in Figs. 19 and 20, respectively.

In Table XI we summarize the results for the critical hopping parameter $K_{c}$ and the masses at $K_{c}$ together with the errors estimated by the least mean square fit and those by the jackknife method. We find that the error estimated by the jackknife method is larger than that by the least mean square fit except for $K_{c}$ at $\beta=6.0$. We take the error obtained by the jackknife method as our estimate of the statistical uncertainty, unless otherwise stated.

\section{Systematic error analyses}

We first estimate the systematic error on the masses in the chiral limit coming from uncertainties in the choice of fitting range for extracting the ground state mass at each $K$. To this end, we repeat linear extrapolations of the masses obtained from the fits to a range $t_{0}-T / 2$, varying $t_{0}$ (common to all
$K^{\prime}$ 's) from $\max _{K}\left\{t_{\chi^{2}}(K)\right\}$ to $t_{\min }+4$. We find that the quality of the linear fits depends on the choice of $t_{0}: \chi^{2} / N_{\mathrm{DF}}$ values are considerably larger for some choices of $t_{0}$. We adopt the condition $\chi^{2} / N_{\mathrm{DF}}<2$ for the linear fit to be accepted. We take the difference between the fitted mass value and the maximum (minimum) mass value under the condition $\chi^{2} / N_{\mathrm{DF}}<2$ as our estimate of the systematic upper (lower) error. We call the systematic error thus obtained the fit-range systematic error.

Data at the fourth largest $K$ slightly deviate from the linear fit. In order to estimate the systematic error which comes from the choice of fitting function, we make a quadratic fit $\left[f(K)=a_{0}+a_{1} / K+a_{2} / K^{2}\right]$ to the largest four $K$ 's, varying $t_{0}$ in the range used for the estimate of the fit-range systematic error. We estimate the systematic error by the difference between the maximum (minimum) value with $\chi^{2} / N_{\mathrm{DF}}<2$ and that of the linear fits. We call the systematic error thus obtained the fit-func. systematic error.

\section{Pion mass extrapolation and $K_{c}$}

Pion masses squared are fitted to a linear function of $1 / K$ to obtain the critical hopping parameter. The value of $\chi^{2} / N_{\mathrm{DF}}$ is $0.56(1.1)$ for the fit $\left[t_{\min }=12(15)\right]$ at $\beta=5.85$ (6.0). The fit-range systematic errors are estimated from the fits with $t_{0}=8-16$ at $\beta=5.85$ and $10-19$ at $\beta=6.0$. All the fits give $\chi^{2} / N_{\mathrm{DF}}<2$. The upper (lower) bound comes from the fit with $t_{0}=11$ (14) with $\chi^{2} / N_{\mathrm{DF}}$ of $0.36(0.04)$ for $\beta=5.85$ and from the fit with $t_{0}=12$ (19) with $\chi^{2} / N_{\mathrm{DF}}$ of 0.44 (0.96) for $\beta=6.0$.

For data at $\beta=5.85$, no quadratic fits with $t_{0}=8-16$ give $\chi^{2} / N_{\mathrm{DF}}<2$. On the other hand, quadratic fits to data at

TABLE X. Fit parameters of the linear fits to the masses at the largest three $K$ 's. Errors on $a_{0}$ and $a_{1}$ are those from least mean square fits.

\begin{tabular}{ccccccc}
\hline \hline & & $\beta=5.85$ & & & $\beta=6.0$ & \\
& $a_{0}$ & $a_{1}$ & $\chi^{2} / N_{\mathrm{DF}}$ & $a_{0}$ & $a_{1}$ & $\chi^{2} / N_{\mathrm{DF}}$ \\
\hline$m_{\pi}^{2}$ & $-7.18(4)$ & $1.16(1)$ & 0.56 & $-6.51(6)$ & $1.02(1)$ & 1.06 \\
$m_{\rho}$ & $-6.16(37)$ & $1.06(6)$ & 1.76 & $-6.50(39)$ & $1.07(6)$ & 1.20 \\
$m_{N}$ & $-10.87(51)$ & $1.85(8)$ & 0.37 & $-12.97(79)$ & $2.11(12)$ & 0.05 \\
$m_{\Delta}$ & $-11.37(80)$ & $1.95(13)$ & 0.05 & $-8.4(1.3)$ & $1.42(21)$ & 0.29 \\
\hline \hline
\end{tabular}




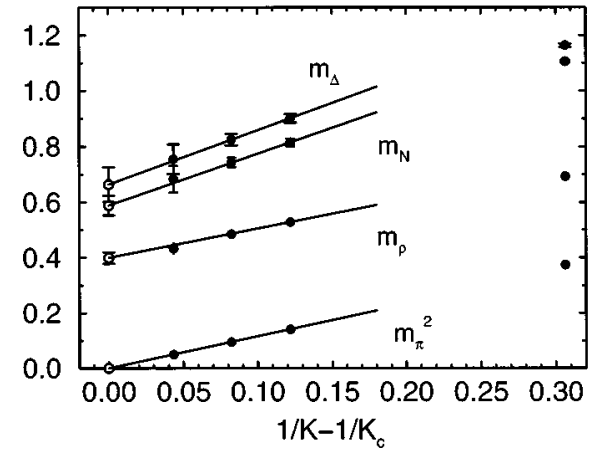

FIG. 19. Linear extrapolations of hadron masses at $\beta=5.85$ to the chiral limit. The open circles at zero quark mass are extrapolated values. The errors shown are statistical only, and do not include the systematic errors discussed in the text.

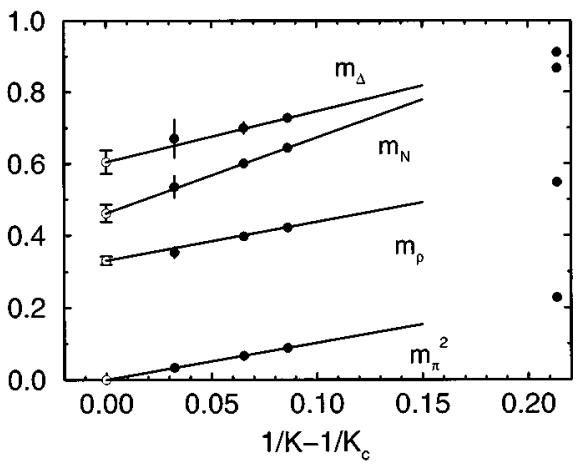

FIG. 20. The same as Fig. 19 for $\beta=6.0$.

TABLE XI. Values of $K_{c}$ and masses extrapolated to $K_{c}$ determined from the linear fits to the data at the largest three $K$ 's. Errors obtained by least mean square fits (err-LMS) and those by the jackknife method (err-jack) together with their ratios (jack/LMS) are also given.

\begin{tabular}{llllc}
\hline \hline & \multicolumn{5}{c}{$\beta=5.85$} & & \\
& Value & err-LMS & err-jack & jack/LMS \\
\hline$K_{c}$ & 0.161624 & 0.000027 & 0.000033 & 1.2 \\
$m_{\rho}\left(K_{c}\right)$ & 0.400 & 0.010 & 0.021 & 2.1 \\
$m_{N}\left(K_{c}\right)$ & 0.589 & 0.014 & 0.036 & 2.6 \\
$m_{\Delta}\left(K_{c}\right)$ & 0.664 & 0.022 & 0.063 & 2.9 \\
\hline & & $\beta=6.0$ & & \\
& Value & err-LMS & err-jack & jack/LMS \\
\hline$K_{c}$ & 0.157096 & 0.000038 & 0.000028 & 0.7 \\
$m_{\rho}\left(K_{c}\right)$ & 0.3309 & 0.0080 & 0.0114 & 1.4 \\
$m_{N}\left(K_{c}\right)$ & 0.462 & 0.015 & 0.024 & 1.6 \\
$m_{\Delta}\left(K_{c}\right)$ & 0.605 & 0.025 & 0.033 & 1.3 \\
\hline \hline
\end{tabular}

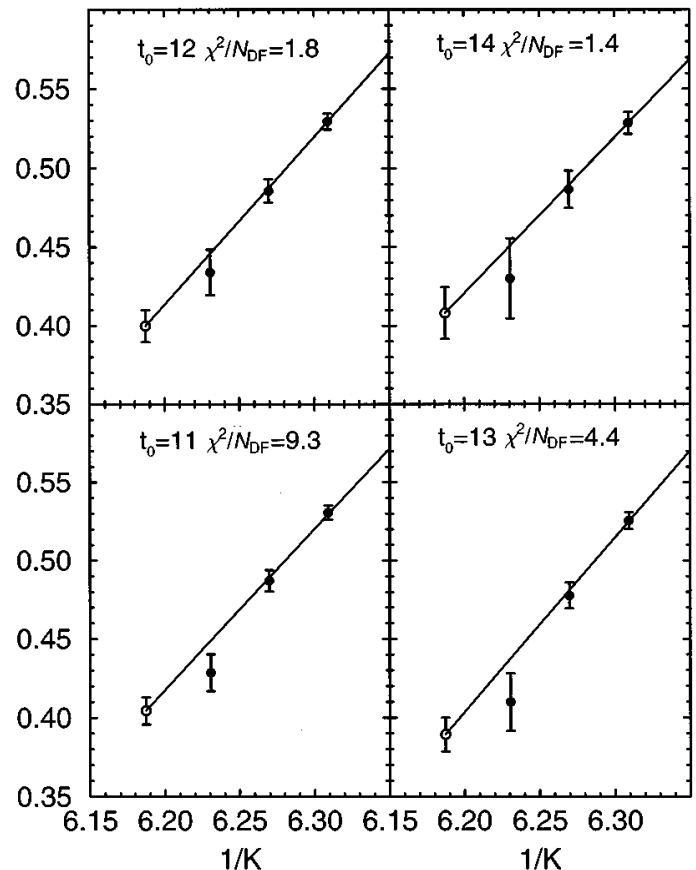

FIG. 21. $\rho$ meson masses at $\beta=5.85$ obtained from the fit with various $t_{0}$ together with linear extrapolations of these data. The open circles are extrapolated values. The errors shown are those estimated by the least mean square fits.

$\beta=6.0$ with $t_{0}=13-19$ give $\chi^{2} / N_{\mathrm{DF}}<2$. Because $m_{\pi}^{2}$ is a concave function of $1 / K$ when the data at the fourth largest $K$ are included, $K_{c}$ obtained from the quadratic fit is larger than that from the linear fit.

The values of $K_{c}$ 's together with the fit-range systematic error and the fit-func. systematic error are given by

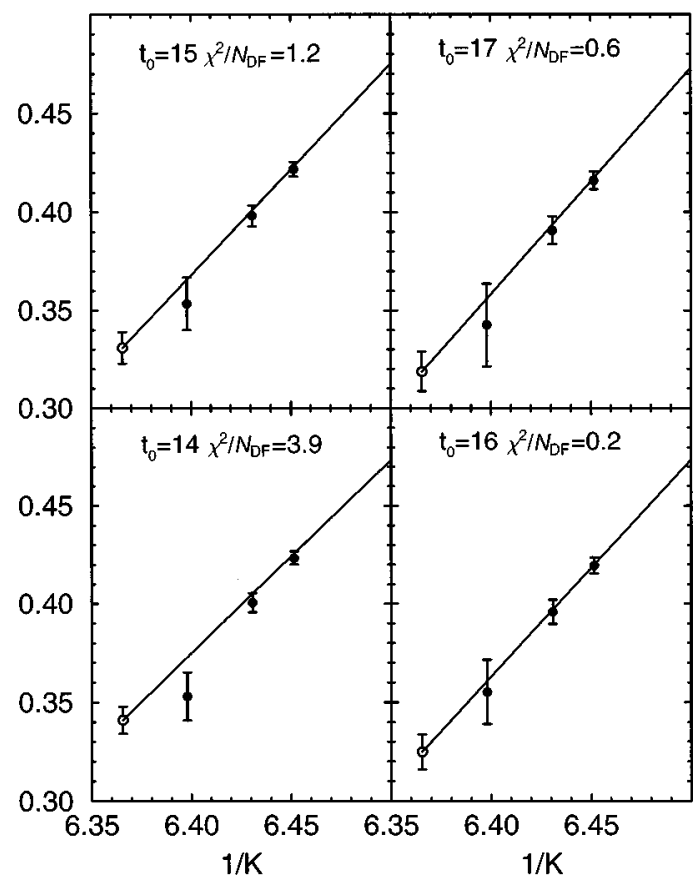

FIG. 22. The same as Fig. 21 but for $\beta=6.0$. 
Stat. $\quad$ Syst. (fit range)

Syst. (fit func.)

\begin{tabular}{llllll}
\hline$\beta=5.85$ & $K_{c}=0.161624$ & \pm 0.000033 & +0.000001 & -0.000025 & \\
$\beta=6.00$ & $K_{c}=0.157096$ & \pm 0.000028 & +0.000033 & -0.000009 & +0.000109
\end{tabular}

The fit-range systematic error is comparable to the statistical uncertainty.

The result for $K_{c}$ at $\beta=6.0$ agrees well with that in Ref. [7]. Although it is slightly smaller than the LANL result 0.157 14(1) [11], we conclude that our result is consistent with theirs within the sum of the statistical error and the fit-range systematic error.

In this work, we do not distinguish the physical point where $m_{\pi} / m_{\rho}$ takes its experimental value from the critical point where the pion mass vanishes, because we find that physical quantities at the two points differ by only at most $30 \%$ of their statistical errors.

\section{E. $\rho$ meson mass extrapolation and lattice spacing}

A linear fit to the $\rho$ meson masses [with $t_{\min }=12$ (15)] at the largest three $K$ 's gives $\chi^{2} / N_{\mathrm{DF}}$ of $1.8(1.2)$ for $\beta=5.85(6.0)$. Therefore the linear fit is acceptable.

However, we find that the quality of the linear fit strongly depends on the choice of fitting range. See Figs. 21 and 22 . In Table XII, we summarize $\chi^{2} / N_{\mathrm{DF}}, m_{\rho}\left(K_{c}\right)$, and the inverse lattice spacing defined by $a^{-1}=(0.77 \mathrm{GeV}) / m_{\rho}\left(K_{c}\right)$ versus $t_{0}$.

We also make a quadratic fit to the data at the largest four $K$ 's to estimate the systematic error due to the choice of fitting function. Table XIII summarizes the results of the quadratic fits versus $t_{0}$.

The method to estimate the systematic error is the same as that adopted for the pion. Our final results for $m_{\rho}$ are

\begin{tabular}{|c|c|c|c|c|c|c|}
\hline & & Stat. & Syst. (fit range) & & st. (fit & \\
\hline$\beta=5.85$ & $m_{\rho}\left(K_{c}\right)=0.400$ & \pm 0.021 & +0.008 & -0.027 & +0.0 & -0.013 \\
\hline$\beta=6.00$ & $m_{\rho}\left(K_{c}\right)=0.331$ & \pm 0.011 & +0.018 & -0.020 & +0.0 & -0.008 \\
\hline
\end{tabular}

The value of $m_{\rho}\left(K_{c}\right)$ at $\beta=6.0$ agrees well with the APE result $0.3332(75)$ and the LANL result $0.3328(106)$. The values of $m_{\rho}\left(K_{c}\right)$ 's are translated to the lattice spacing as

\begin{tabular}{|c|c|c|c|c|c|c|}
\hline & & Stat. & Syst. (fit range) & & yst. (fit $f$ & \\
\hline$\beta=5.85$ & $a^{-1}=1.93$ & \pm 0.10 & +0.14 & -0.04 & +0.08 & $-0.0 \mathrm{GeV}$ \\
\hline$\beta=6.00$ & $a^{-1}=2.33$ & \pm 0.08 & +0.15 & -0.12 & +0.06 & $-0.0 \mathrm{GeV}$ \\
\hline
\end{tabular}

Although the statistical error on $a^{-1}$ is several percent, we notice that the systematic error is much larger. Summing up both the statistical and systematic errors, we find that $a^{-1}$ can be as large as $2.25 \mathrm{GeV}(2.62 \mathrm{GeV})$ at $\beta=5.85(6.0)$ and as small as $1.79 \mathrm{GeV}(2.13 \mathrm{GeV})$.

In analyses of the systematic errors above, we have taken

TABLE XII. Results of the linear fits to the $\rho$ meson masses versus $t_{0}$. The inverse lattice spacing is defined by $a^{-1}=(0.77 \mathrm{GeV}) / m_{\rho}\left(K_{c}\right)$.

\begin{tabular}{rccccccc}
\hline \hline \multicolumn{4}{c}{$\beta=5.85$} & \multicolumn{4}{c}{$\beta=6.0$} \\
\multicolumn{1}{c}{$t_{0}$} & $m_{\rho}$ & $a^{-1}$ & $\chi^{2} / N_{\mathrm{DF}}$ & \multicolumn{1}{c}{$t_{0}$} & $m_{\rho}$ & $a^{-1}$ & $\chi^{2} / N_{\mathrm{DF}}$ \\
\hline 8 & 0.4359 & 1.766 & 36.90 & 11 & 0.3525 & 2.184 & 2.51 \\
9 & 0.4213 & 1.828 & 11.17 & 12 & 0.3476 & 2.215 & 2.54 \\
10 & 0.4196 & 1.835 & 14.80 & 13 & 0.3425 & 2.248 & 0.77 \\
11 & 0.4045 & 1.904 & 9.30 & 14 & 0.3409 & 2.259 & 3.94 \\
12 & 0.3998 & 1.926 & 1.76 & 15 & 0.3309 & 2.327 & 1.20 \\
13 & 0.3892 & 1.978 & 4.39 & 16 & 0.3248 & 2.370 & 0.17 \\
14 & 0.4081 & 1.887 & 1.36 & 17 & 0.3188 & 2.416 & 0.58 \\
15 & 0.3794 & 2.030 & 0.43 & 18 & 0.3112 & 2.474 & 0.03 \\
16 & 0.3728 & 2.066 & 0.11 & 19 & 0.3206 & 2.401 & 0.00 \\
\hline \hline
\end{tabular}

$t_{0}$ common to all $K$ 's. However, it is not necessary to restrict ourselves to taking a common value of $t_{0}$, because the time slice at which the contribution of excited states becomes negligible can depend on the quark mass. We make linear fits to all possible combinations of the $\rho$ masses at the largest three $K$ 's, varying $t_{0}$ separately for each $K$ from $t_{\chi^{2}}$ to 18 . Figure 23 shows $a^{-1}$ at $\beta=6.0$ versus $\chi^{2} / N_{\mathrm{DF}}$. We see that there are linear fits with small $\chi^{2} / N_{\mathrm{DF}}$ which give both large and

TABLE XIII. Results of the quadratic fits to the $\rho$ meson masses versus $t_{0}$. The inverse lattice spacing is defined by $a^{-1}=(0.77 \mathrm{GeV}) / m_{\rho}\left(K_{c}\right)$.

\begin{tabular}{cccccccc}
\hline \hline \multicolumn{3}{c}{$\beta=5.85$} & & \multicolumn{4}{c}{$\beta=6.0$} \\
$t_{0}$ & $m_{\rho}$ & $a^{-1}$ & $\chi^{2} / N_{\mathrm{DF}}$ & $t_{0}$ & $m_{\rho}$ & $a^{-1}$ & $\chi^{2} / N_{\mathrm{DF}}$ \\
\hline 12 & 0.3881 & 1.984 & 1.22 & 13 & 0.3413 & 2.256 & 0.79 \\
13 & 0.3767 & 2.044 & 4.02 & 14 & 0.3393 & 2.269 & 4.33 \\
14 & 0.3997 & 1.927 & 1.20 & 15 & 0.3253 & 2.367 & 0.91 \\
15 & 0.3641 & 2.115 & 0.21 & 16 & 0.3194 & 2.411 & 0.07 \\
16 & 0.3593 & 2.143 & 0.30 & 17 & 0.3116 & 2.471 & 0.41 \\
& & & & 18 & 0.3029 & 2.542 & 0.00 \\
& & & & 19 & 0.3146 & 2.448 & 0.01 \\
\hline \hline
\end{tabular}


small $a^{-1}$. The value of $a^{-1}$ scatters approximately from $2.15 \mathrm{GeV}$ to $2.65 \mathrm{GeV}$. This upper value as well as the lower value are consistent with those obtained above with the systematic errors included.

We estimate the value of $J$ defined by $m_{V} d m_{V} / d m_{P}^{2}$ [16] from the linear fits discussed above:

\begin{tabular}{ccccc}
\multicolumn{4}{c}{ Stat. } & Syst. (fit range) \\
\hline$\beta=5.85$ & $J=0.420$ & \pm 0.049 & +0.028 & -0.024 \\
$\beta=6.00$ & $J=0.395$ & \pm 0.026 & +0.026 & -0.026
\end{tabular}

The value of $J$ at $\beta=6.0$ is smaller than the experimental value $0.48(2)$ even when we include the systematic errors.

\section{F. Nucleon and $\Delta$ masses}

Both linear fits and quadratic fits are made to the masses of the nucleon and the $\Delta$ baryon by the same method as for the $\rho$ meson. Results of the linear fits versus the fit range are summarized in Tables XIV and XV. The fit with $t_{\min }=13(16)$ at $\beta=5.85(6.0)$, which is adopted in this work, gives a small $\chi^{2} / N_{\mathrm{DF}}=0.37(0.05)$. For the nucleon, the quality of the linear fits is good for almost all values of $t_{0}$ in the sense that $\chi^{2} / N_{\mathrm{DF}}$ is approximately less than 2, except for the fit with $t_{0}=9$ at $\beta=5.85$. This feature is different from that for the $\rho$ meson. The quality of the fits to the $\Delta$ masses at $\beta=5.85$ is good for $t_{0} \leqslant 13$ including our choice $t_{\min }=13$ and that at $\beta=6.0$ is good for all $t_{0}$ except for $t_{0}=13$.

Results with various errors are given by

\begin{tabular}{|c|c|c|c|c|c|c|}
\hline & & Stat. & Syst. (fit range) & & yst. (fit f & \\
\hline$\beta=5.85$ & $m_{N}\left(K_{c}\right)=0.589$ & \pm 0.036 & +0.018 & -0.058 & +0.0 & -0.018 \\
\hline$\beta=6.00$ & $m_{N}\left(K_{c}\right)=0.462$ & \pm 0.024 & +0.020 & -0.009 & +0.0 & -0.007 \\
\hline$\beta=5.85$ & $m_{\Delta}\left(K_{c}\right)=0.664$ & \pm 0.063 & +0.034 & -0.0 & +0.0 & -0.031 \\
\hline$\beta=6.00$ & $m_{\Delta}\left(K_{c}\right)=0.605$ & \pm 0.033 & +0.041 & -0.011 & +0.016 & -0.007 \\
\hline
\end{tabular}

The value of the nucleon mass in the chiral limit at $\beta=6.0$ lies between the LANL result $0.482(13)$ and the APE result $0.432(15)$. For the $\Delta$ masses, results by the three groups agree well with each other, albeit with large errors; the LANL result is 0.590(30) and the APE result 0.58(3). The LANL results are those at the physical point where $m_{\pi} / m_{\rho}$ takes its experimental value.

These results are translated to the masses in physical units using the value of $a^{-1}$ obtained from $m_{\rho}$. The systematic error on the lattice spacing is not taken into account for the estimate of the systematic error on the baryon masses. The results read

\begin{tabular}{|c|c|c|c|c|c|c|}
\hline & & Stat. & Syst. (fit range) & & yst. (fit f & \\
\hline$\beta=5.85$ & $m_{N}=1.135$ & \pm 0.088 & +0.034 & -0.112 & +0.0 & $-0.034 \mathrm{GeV}$ \\
\hline$\beta=6.00$ & $m_{N}=1.076$ & \pm 0.060 & +0.047 & -0.020 & +0.0 & $-0.017 \mathrm{GeV}$ \\
\hline$\beta=5.85$ & $m_{\Delta}=1.279$ & \pm 0.136 & +0.066 & -0.0 & +0.0 & $-0.059 \mathrm{GeV}$ \\
\hline$\beta=6.00$ & $m_{\Delta}=1.407$ & \pm 0.086 & +0.096 & -0.026 & +0.038 & $-0.015 \mathrm{GeV}$ \\
\hline
\end{tabular}

The central value of the nucleon mass at $\beta=6.0(5.85)$ is larger than its experimental value by about $15 \%(20 \%)$ and that of the $\Delta$ mass by about $15 \%$ (4\%): The errors amount to twice the statistical errors except for the $\Delta$ baryon at $\beta=5.85$. The systematic errors are comparable with the statistical errors $(3-13 \%)$. Even when the systematic errors are included, the baryon masses at $\beta=6.0$ do not agree with experiment. Our data are consistent with the GF11 data [10] at finite lattice spacing, within statistical errors. In order to take the continuum limit of our results, we need data for a wider range of $\beta$ with statistical and systematic errors much reduced.

\section{G. Masses of strange hadrons}

The hopping parameters for the strange quark which are estimated from the experimental value of $m_{K} / m_{\rho}$ turn out to be $K_{s}=0.1588$ and 0.1550 at $\beta=5.85$ and 6.0 , respectively.
Note that they are identical or almost identical to the values of the third largest hopping parameter $K=0.1585$ and 0.1550 which we have chosen in such a way that they approximately correspond to the strange quark. The masses of $\Omega^{-}$estimated at $K=K_{s}$ are $1.696(92) \mathrm{GeV}$ and $1.693(57) \mathrm{GeV}$ at $\beta=5.85$ and 6.0, respectively (statistical errors only). They are in good agreement with the experimental value 1.672 $\mathrm{GeV}$. The masses of the vector meson at $K=K_{s}$ are 998(45) $\mathrm{MeV}$ and $986(26) \mathrm{MeV}$ at $\beta=5.85$ and 6.0, respectively, which equal the $\phi$ meson mass $1019 \mathrm{MeV}$ within about one standard deviation. As is well known, there are ambiguities in determination of the hopping parameter for the strange quark. When the hopping parameters for the strange quark mass are alternatively determined from $m_{\phi} / m_{\rho}$, they are equal to 0.1585 and 0.1547 . The results for the $\Omega^{-}$mass at these hopping parameters are consistent with those above within one standard deviation. 
TABLE XIV. Results of the linear fits to the nucleon masses versus $t_{0}$.

\begin{tabular}{rccccc}
\hline \hline & $\beta=5.85$ & & \multicolumn{3}{c}{$\beta=6.0$} \\
\multicolumn{1}{c}{$t_{0}$} & $m_{N}$ & $\chi^{2} / N_{\mathrm{DF}}$ & $t_{0}$ & $m_{N}$ & $\chi^{2} / N_{\mathrm{DF}}$ \\
\hline 9 & 0.6085 & 3.20 & 12 & 0.4828 & 0.03 \\
10 & 0.6071 & 0.37 & 13 & 0.4802 & 0.24 \\
11 & 0.6039 & 0.83 & 14 & 0.4758 & 0.79 \\
12 & 0.5946 & 2.15 & 15 & 0.4759 & 1.88 \\
13 & 0.5893 & 0.37 & 16 & 0.4623 & 0.05 \\
14 & 0.5680 & 2.28 & 17 & 0.4538 & 2.00 \\
15 & 0.5501 & 1.59 & 18 & 0.4553 & 2.28 \\
16 & 0.5312 & 0.49 & 19 & 0.4731 & 0.10 \\
17 & 0.5630 & 2.55 & 20 & 0.4559 & 0.10 \\
\hline \hline
\end{tabular}

\section{MESON DECAY CONSTANTS}

\section{A. Vector meson decay constants}

We evaluate vector meson decay constants defined by

$$
\left\langle 0\left|\left(\bar{u} \gamma_{i} d\right)^{\mathrm{cont}}\right| V(\vec{p}=0)\right\rangle=\epsilon_{i} F_{V} m_{V},
$$

where $\epsilon_{i}$ and $m_{V}$ are the polarization vector and the mass of the vector meson, respectively, and $\left(\bar{u} \gamma_{i} d\right)^{\text {cont }}$ is the vector current in the continuum limit. The experimental value for the $\rho$ meson is $F_{\rho}=216(5) \mathrm{MeV}$. (This $F_{V}$ is related to $f_{V}^{-1}$ by $f_{V}^{-1}=F_{V} / m_{V}$.)

The expectation value of the local lattice current $\left(\bar{u} \gamma_{i} d\right)^{\text {latt }}$ between the vacuum and the vector meson is related to the continuum one by the relation

$$
\left\langle 0\left|\left(\bar{u} \gamma_{i} d\right)^{\mathrm{cont}}\right| V(\vec{p}=0)\right\rangle=Z_{K} Z_{V}\left\langle 0\left|\left(\bar{u} \gamma_{i} d\right)^{\mathrm{latt}}\right| V(\vec{p}=0)\right\rangle .
$$

The coefficient $Z_{K}$ is a scale factor for the difference between the continuum and lattice normalizations of the quark field. The renormalization constant $Z_{V}$ is the ratio of the conserved lattice current to the local current, which can be estimated by perturbation theory or numerical simulations. We test the following three possible choices of $Z_{K}$ and $Z_{V}$.

(1) Those in naive perturbation theory: $Z_{K}=2 K$ and $Z_{V}=1-0.174 g^{2}[19]$.

(2) Those in tadpole improved perturbation theory: $Z_{K}=\left(1-3 K / 4 K_{c}\right) \quad[20]$ and $Z_{V}=1-0.82 \alpha \overline{\mathrm{MS}}(1 / a)$ [21],

TABLE XV. Results of the linear fits to the $\Delta$ masses versus $t_{0}$.

\begin{tabular}{lccccc}
\hline \hline & $\beta=5.85$ & & \multicolumn{3}{c}{$\beta=6.0$} \\
$t_{0}$ & $m_{\Delta}$ & $\chi^{2} / N_{\mathrm{DF}}$ & $t_{0}$ & $m_{\Delta}$ & $\chi^{2} / N_{\mathrm{DF}}$ \\
\hline 11 & 0.6982 & 0.03 & 12 & 0.6055 & 0.01 \\
12 & 0.6928 & 0.01 & 13 & 0.6059 & 4.10 \\
13 & 0.6640 & 0.05 & 14 & 0.6164 & 1.78 \\
14 & 0.6899 & 4.02 & 15 & 0.6279 & 0.50 \\
15 & 0.5375 & 34.96 & 16 & 0.6048 & 0.29 \\
16 & 0.4757 & 23.96 & 17 & 0.6206 & 0.12 \\
& & & 18 & 0.6462 & 0.17 \\
& & & 19 & 0.5935 & 0.34 \\
\hline \hline
\end{tabular}

TABLE XVI. Renormalization constants $Z_{V}$ for the local lattice current at $\beta=5.85$ obtained in a previous work [2].

\begin{tabular}{cl}
\hline \hline$K$ & \multicolumn{1}{c}{$Z_{V}$} \\
\hline 0.1440 & $0.5121(9)$ \\
0.1540 & $0.5164(10)$ \\
0.1585 & $0.5126(36)$ \\
0.1595 & $0.5112(48)$ \\
0.1605 & $0.5101(76)$ \\
Average & $0.5125(30)$ \\
\hline
\end{tabular}

where $\overline{\mathrm{MS}}$ is the modified minimal subtraction scheme. $\left[\alpha_{\overline{\mathrm{MS}}}(\pi / a)=g_{\overline{\mathrm{MS}}}^{2}(\pi / a) / 4 \pi\right.$ is determined by the relation $1 / g_{\overline{\mathrm{MS}}}^{2}(\pi / a)=\operatorname{Tr}\left(U_{P} / 3\right) / g^{2}+0.02461[21,22]$. We then determine $\alpha_{\overline{\mathrm{MS}}}(1 / a)$ using the two-loop renormalization group equation.]

(3) Monte Carlo estimate of $Z_{V}=0.51$ [2] (0.57 [23]) at $\beta=5.85$ (6.0) with $Z_{K}=2 K$. (Data for $Z_{V}$ at $\beta=5.85$ [2] are given in Table XVI. Because the results for $Z_{V}$ are independent of the quark mass in the range we investigate, we use the averaged value.) The error on $Z_{V}$ is ignored in the following.

We abbreviate the decay constants obtained using the above three renormalization constants as $F_{V}^{\mathrm{PT}}, F_{V}^{\mathrm{TP}}$, and $F_{V}^{\mathrm{MC}}$, respectively.

The statistical error is obtained by the jackknife method. The systematic error is estimated varying $t_{0}$ as in the case of the mass calculation. The range of $t_{0}$ is the same as that for the $\rho$ mass. In Table XVII we summarize the results for the decay constants at each $K$. We quote the error only for $F_{V}^{\mathrm{TP}}$, because the errors for the others can be easily obtained from that for $F_{V}^{\mathrm{TP}}$ by multiplying the ratio of $Z$ factors.

Figure 24 shows $F_{V} / m_{V}$ versus $\left(m_{P} / m_{V}\right)^{2}$ together with the corresponding experimental values for $\rho, \omega, \phi$, and $J / \psi$. Note that we can compare the numerical results with the experimental values for $\phi$ and $J / \psi$ without extrapolation. The values with $F_{V}^{\mathrm{MC}}$ at the two $\beta$ 's remarkably agree with each other. Furthermore, they agree well with the experimental values for $\phi$ and $J / \psi$. This implies that scaling violation in $F_{V}^{\mathrm{MC}}$ is small. On the other hand, we find sizable scaling

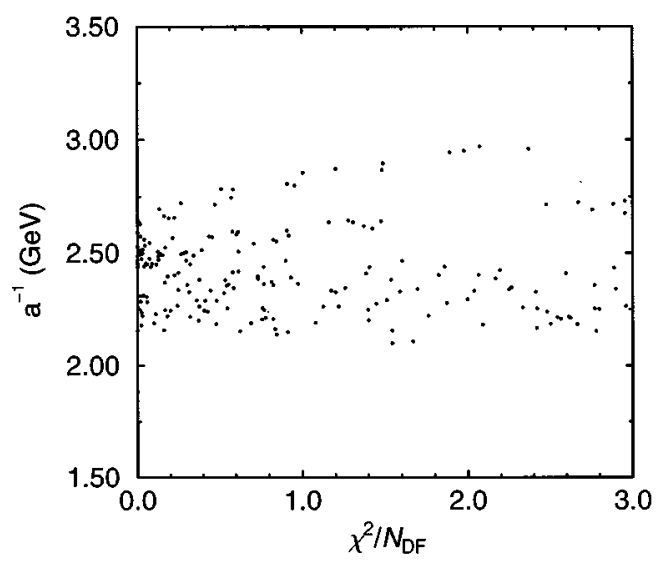

FIG. 23. $a^{-1}$ determined from linear fits to all possible combinations of the $\rho$ masses obtained by varying $t_{0}$ from $t_{\chi^{2}}$ to 18 . 
TABLE XVII. $\rho$ meson decay constants in lattice units. In parentheses are errors estimated by the jackknife method. Errors given in the form ${ }_{-}^{+}$lower are for the fitting range dependent upper (lower) bounds.

\begin{tabular}{cccccccc}
\hline \hline & \multicolumn{3}{c}{$\beta=5.85$} & & \multicolumn{3}{c}{$\beta=6.0$} \\
$K$ & $F_{V}^{\mathrm{PT}}$ & $F_{V}^{\mathrm{TP}}$ & $F_{V}^{\mathrm{MC}}$ & $K$ & $F_{V}^{\mathrm{PT}}$ & $F_{V}^{\mathrm{TP}}$ & $F_{V}^{\mathrm{MC}}$ \\
\hline 0.1440 & 0.2214 & $0.2600(33)_{-0}^{+17}$ & 0.1374 & 0.1450 & 0.1753 & $0.1919(18)_{-13}^{+5}$ & 0.1210 \\
0.1540 & 0.2211 & $0.2089(38)_{-17}^{+5}$ & 0.1372 & 0.1520 & 0.1673 & $0.1558(17)_{-19}^{+14}$ & 0.1155 \\
0.1585 & 0.2094 & $0.1781(68)_{-130}^{+126}$ & 0.1299 & 0.1550 & 0.1544 & $0.1336(33)_{-78}^{+65}$ & 0.1065 \\
0.1595 & 0.1996 & $0.1658(84)_{-142}^{+162}$ & 0.1239 & 0.1555 & 0.1493 & $0.1276(43)_{-88}^{+106}$ & 0.1031 \\
0.1605 & 0.1885 & $0.1528(152)_{-201}^{+153}$ & 0.1170 & 0.1563 & 0.1382 & $0.1157(95)_{-87}^{+181}$ & 0.0953 \\
\hline \hline
\end{tabular}

violation in $F_{V}^{\mathrm{PT}}$ and $F_{V}^{\mathrm{TP}}$. They are off the experimental values for $\phi$ and $J / \psi$ by $40-100 \%$. We find that the $F_{V}^{\mathrm{MC}} / m_{V}$ 's at $\beta=6.0$ agree well with the APE data $[7,8]$.

In Fig. 25 we depict the values of $F_{\phi} / m_{\rho}$ versus $m_{\rho} a$ together with the GF11 result [24]. The values of the hopping parameter for the strange quark are given in Sec. V G. Note that the values of $F_{\phi}^{\mathrm{MC}} / m_{\rho}$ agree with experiment already at $m_{\rho} a=0.33-0.40$ within $1-2$ standard deviations. The values of $F_{\phi}^{\mathrm{TP}} / m_{\rho}$ are consistent with the GF11 result, although the central values are about $1 \sigma$ higher than the GF11 data. They are off the experimental value by $30-40 \%$ at these values of $m_{\rho} a$. Linear extrapolation of our data to zero lattice spacing is consistent with experiment.

The value of $F_{V}$ in the chiral limit is obtained from a linear fit in terms of $1 / K$ in a similar way to that made for hadron mass extrapolation. We first calculate the correlation matrix $\Sigma\left(K, K^{\prime}\right)$ for $F_{V}(K)$ from the error matrix $\Sigma$ for the mass and amplitude [Eq. (15)] using the error propagation rule, and then minimize $\chi^{2}$. A linear fit to the data at the largest three $K$ 's gives a reasonable $\chi^{2} / N_{\mathrm{DF}}: \chi^{2} / N_{\mathrm{DF}}=0.04$ $(0.38)$ for $F_{V}^{\mathrm{TP}}$ and $0.09(0.44)$ for $F_{V}^{\mathrm{PT}}$ and $F_{V}^{\mathrm{MC}}$ at $\beta=5.85$ (6.0), respectively. Figure 26 shows $F_{V}$ as a function of the quark mass together with the fitting functions.

The method to estimate the systematic error due to the choice of fitting range is similar to that for hadron masses at $K_{c}$. The results of the linear fit for various fitting ranges are given in Table XVIII. Our final results for $F_{\rho}$ read

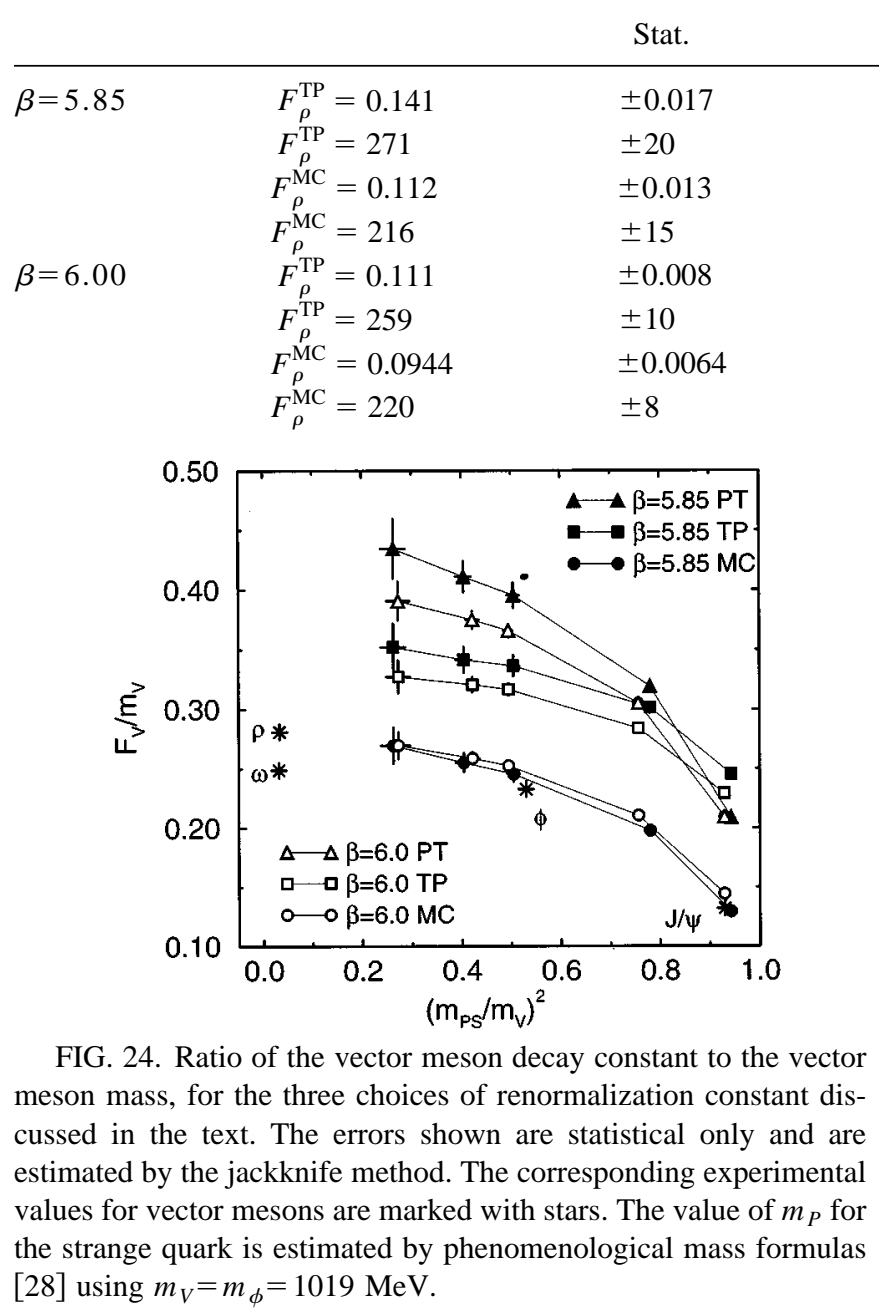

Syst. (fit range)

$\begin{array}{ll}+0.007 & -0.035 \\ +14 & -68 \mathrm{MeV} \\ +0.006 & -0.027 \\ +11 & -52 \mathrm{MeV} \\ +0.016 & -0.017 \\ +37 & -40 \mathrm{MeV} \\ +0.010 & -0.014 \\ +24 & -33 \mathrm{MeV}\end{array}$

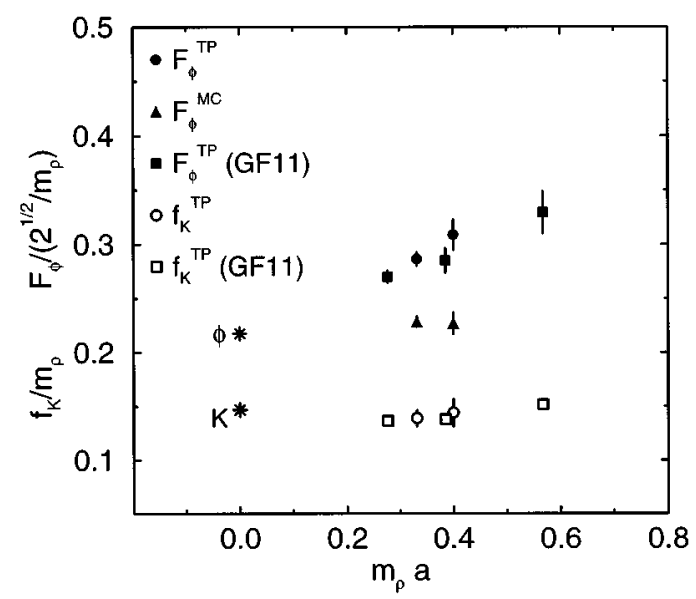

FIG. 25. Ratios of the $\phi$ meson decay constant and the $K$ meson decay constant to the $\rho$ meson mass versus the $\rho$ meson mass in lattice units. The errors in our data are statistical only. The GF11 data are taken from Ref. [24]. The corresponding experimental values are marked with stars. 
TABLE XVIII. Results of the linear fits to the $\rho$ meson decay constants versus $t_{0}$, in lattice units and in physical units $(\mathrm{MeV})$.

\begin{tabular}{|c|c|c|c|c|c|c|c|c|c|c|c|c|c|}
\hline & & & $\beta=5$. & & & & & & & $\beta=\epsilon$ & & & \\
\hline & & $F_{V}^{\mathrm{TP}}$ & & & $F_{V}^{\mathrm{MC}}$ & & & & F & $\begin{array}{l}\mathrm{TP} \\
V\end{array}$ & & $F_{V}^{\mathrm{MC}}$ & \\
\hline$t_{0}$ & Latt. & Phys. & $\chi^{2} / N_{\mathrm{DF}}$ & Latt. & Phys. & $\chi^{2} / N_{\mathrm{DF}}$ & $t_{0}$ & Latt. & Phys. & $\chi^{2} / N_{\mathrm{DF}}$ & Latt. & Phys. & $\chi^{2} / N_{\mathrm{DF}}$ \\
\hline 8 & 0.169 & 325 & 53.7 & 0.133 & 257 & 55.7 & 11 & 0.127 & 297 & 1.9 & 0.107 & 250 & 2.0 \\
\hline 9 & 0.157 & 303 & 6.0 & 0.125 & 240 & 7.0 & 12 & 0.124 & 289 & 1.7 & 0.105 & 244 & 1.9 \\
\hline 10 & 0.156 & 301 & 11.1 & 0.124 & 239 & 11.9 & 13 & 0.120 & 280 & 0.1 & 0.102 & 237 & 0.2 \\
\hline 11 & 0.145 & 280 & 5.8 & 0.115 & 222 & 6.3 & 14 & 0.120 & 278 & 3.4 & 0.101 & 235 & 3.6 \\
\hline 12 & 0.141 & 271 & 0.0 & 0.112 & 216 & 0.1 & 15 & 0.111 & 259 & 0.4 & 0.094 & 220 & 0.4 \\
\hline 13 & 0.130 & 251 & 2.3 & 0.104 & 201 & 2.6 & 16 & 0.107 & 248 & 0.0 & 0.091 & 211 & 0.0 \\
\hline 14 & 0.148 & 286 & 0.3 & 0.118 & 226 & 0.3 & 17 & 0.101 & 234 & 0.1 & 0.085 & 199 & 0.1 \\
\hline 15 & 0.116 & 224 & 0.0 & 0.094 & 180 & 0.0 & 18 & 0.094 & 220 & 0.1 & 0.080 & 187 & 0.1 \\
\hline 16 & 0.105 & 203 & 1.1 & 0.085 & 164 & 1.1 & 19 & 0.104 & 243 & 0.1 & 0.088 & 206 & 0.1 \\
\hline
\end{tabular}

The values of $F_{\rho}^{\mathrm{PT}}$ can be obtained from $F_{\rho}^{\mathrm{MC}}$ by multiplying $Z_{V}^{\mathrm{PT}} / Z_{V}^{\mathrm{MC}}=1.61(1.45)$ at $\beta=5.85(6.0)$. We show the values of $F_{\rho} / m_{\rho}$ in Fig. 27. It should be noted that the values of $F_{V}^{\mathrm{MC}}$ in the chiral limit at the two $\beta$ 's are consistent with the experimental value of $F_{\rho}$. We find that our values of $F_{\rho}^{\mathrm{TP}} / m_{\rho}$ are consistent with the GF11 result [24], albeit the central values are roughly $1 \sigma$ lower than the GF11 data; this tendency is opposite to the case of the $\phi$ meson. We note that linear extrapolation of our data for $F_{\rho}^{\mathrm{TP}} / m_{\rho}$ to zero lattice spacing is again consistent with experiment.

\section{B. Pseudoscalar meson decay constants}

The pseudoscalar meson decay constant is defined by

$$
\left\langle 0\left|\left(\bar{u} \gamma_{0} \gamma_{5} d\right)^{\mathrm{cont}}\right| P(\vec{p}=0)\right\rangle=\sqrt{2} m_{P} f_{P} .
$$

The experimental value is $f_{\pi}=93 \mathrm{MeV}$. We investigate three cases of renormalization constants as in the case of $F_{V}$ : (1) $Z_{A}=1-0.133 g^{2}$ in naive perturbation theory [19] with $Z_{K}=2 K, \quad$ (2) $Z_{A}=1-0.31 \alpha_{\overline{\mathrm{MS}}}(1 / a) \quad$ [21] with

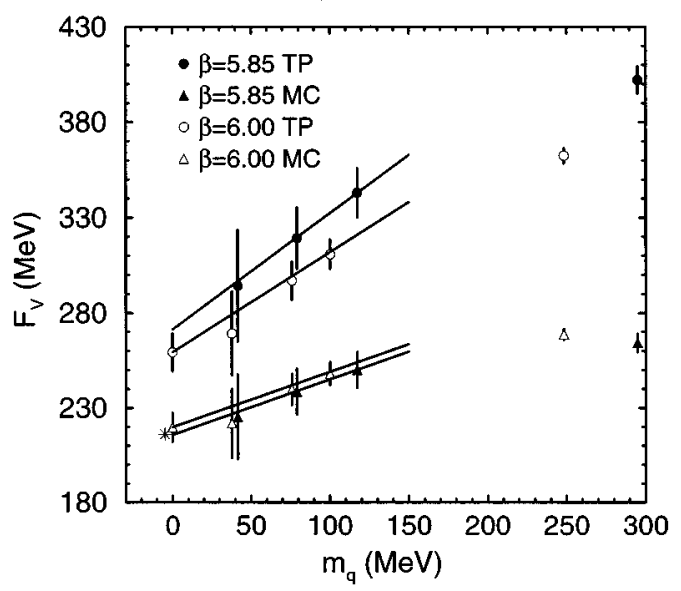

FIG. 26. Linear extrapolations of vector meson decay constant, for the two choices of renormalization constant discussed in the text. The open symbols at zero quark mass are extrapolated values for $\beta=6.0$. The errors shown are statistical only. The experimental value for the $\rho$ meson is marked with a star.
$Z_{K}=\left(1-3 K / 4 K_{c}\right)[20]$ in tadpole improved perturbation theory, and (3) $Z_{A}=0.69$ [23] at $\beta=6.0$ as a nonperturbative evaluation with $Z_{K}=2 K$. (The corresponding $Z_{A}$ at $\beta=5.85$ is not known.)

We derive $f_{P}$ from a fit to the $\widetilde{\pi}$ propagator. The value of $t_{\min }$ is chosen to be the same as that for $\pi$. The pion mass from the $\widetilde{\pi}$ propagator is given in Table XIX. Although the mass obtained is 1-2 standard deviations smaller than that from the $\pi$ propagator, they are consistent with each other if we take account of the systematic error. The decay constant at each $K$ is given in Table XX. Our data for $f_{P}^{\mathrm{PT}}$ at $\beta=6.0$ and $K=0.155,0.1563$ are consistent with the APE results $[7,8]$. Figure 28 shows $f_{P} / m_{V}$ versus $\left(m_{P} / m_{V}\right)^{2}$ together with the corresponding experimental values for $\pi$ and $K$ and the upper bound for the $D$ meson. Contrary to the case of the vector meson, $f_{P}^{\mathrm{MC}}$ differs from the experimental value for the $K$ meson by a factor of about 1.2. There is a possibility that the lattice size $10^{3} \times 20$ is not large enough to suppress finite lattice size effects in the Monte Carlo evaluation of $Z_{A}$. We think we have to calculate $Z_{A}$ nonperturbatively at both $\beta=5.85$ and 6.0 on a larger lattice in order to clarify the reason for the discrepancy.

In Fig. 25 we show the values of $f_{K}^{\mathrm{TP}} / m_{\rho}$ versus $m_{\rho} a$ together with the GF11 result [24]. The values of $f_{K}$ are evaluated at the hopping parameter given by

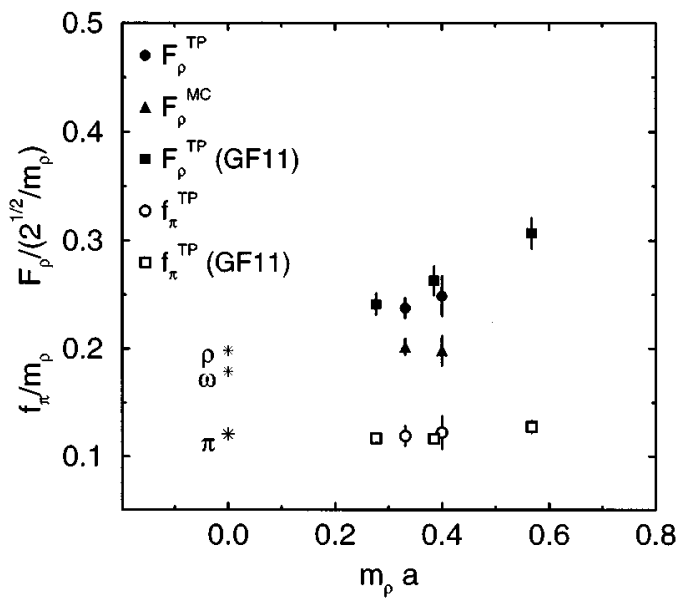

FIG. 27. The same as Fig. 25 for the $\rho$ meson and the pion. 


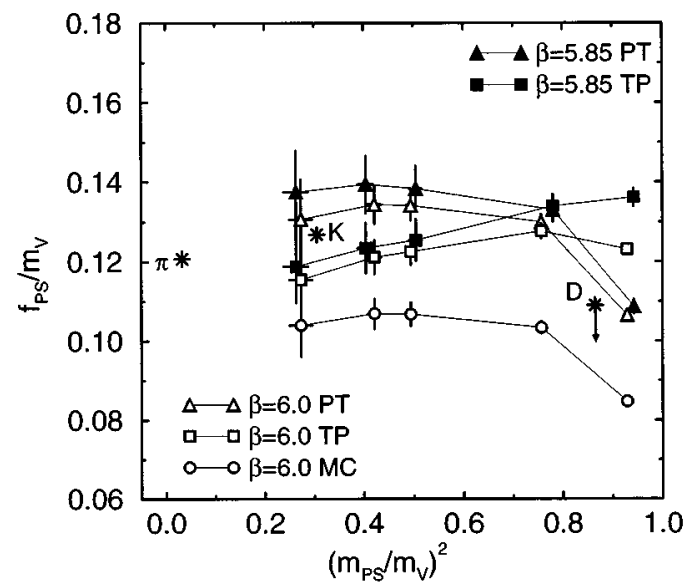

FIG. 28. Ratio of the pseudoscalar meson decay constant to the vector meson mass, for the three choices of renormalization constant discussed in the text. The errors shown are statistical only and are estimated by the jackknife method. The corresponding experimental values for pseudoscalar mesons are marked with stars.

$2 /\left(1 / K_{c}+1 / K_{s}\right)$. The values of $f_{K}^{\mathrm{TP}} / m_{\rho}$ are consistent with the GF11 result, albeit with larger errors in our results. Our data at finite lattice spacing are also consistent with experiment.

The extrapolation to the chiral limit is problematic. We find that neither the linear fit to the data at the three largest $K$ 's nor the quadratic fit to the data at the four largest $K$ 's gives $\chi^{2} / N_{\mathrm{DF}}$ small enough: For $f_{P}^{\mathrm{TP}}$ at $\beta=5.85$ (6.0), $\chi^{2} / N_{\mathrm{DF}}=9.1$ (6.7) for the linear fit and 9.9 (7.4) for the quadratic fit, respectively. Fits to $f_{P}^{\mathrm{MC}}$ are similar. In Fig. 29 are shown $f_{P}$ versus the quark mass together with the linear

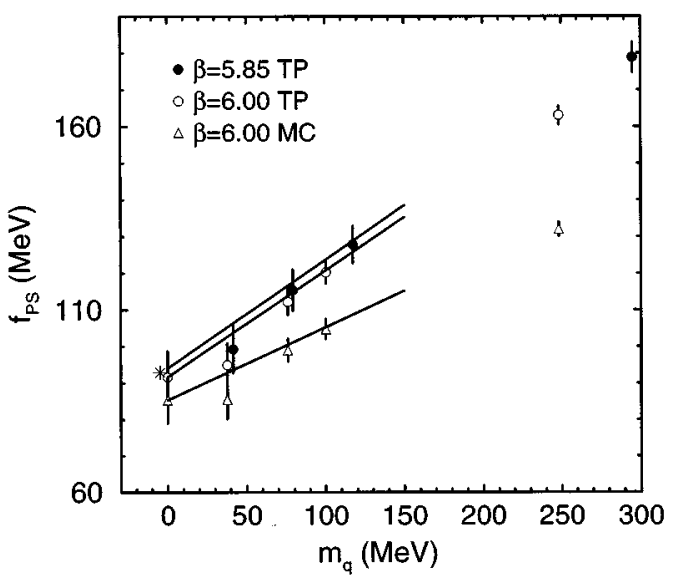

FIG. 29. Linear extrapolations of the pseudoscalar meson decay constant, for the two choices of renormalization constant discussed in the text. The open symbols at zero quark mass are extrapolated values for $\beta=6.0$. The errors shown are statistical only. The experimental value for the pion is marked with a star.

fits. The data at the largest $K$ are much below the fitting lines. Even if we change $t_{\min }, \chi^{2} / N_{\mathrm{DF}}$ is not reduced much. In Fig. 30 we show $\chi^{2} / N_{\mathrm{DF}}$ together with the result for $f_{P}^{\mathrm{TP}}$ at $\beta=6.0$ versus $t_{\min }$. Although $\chi^{2} / N_{\mathrm{DF}}$ is large, the results of the fits are very stable. Therefore we quote the decay constant obtained by the linear extrapolation of the data with $t_{\min }=12(15)$ at $\beta=5.85(6.0)$ as the central value of the decay constant. We estimate the systematic errors similarly as in the previous cases with $t_{0}=t_{\chi^{2}}-14$ (16) for $\beta=5.85$ (6.0).

These analyses give

\begin{tabular}{lllllll}
\multicolumn{3}{c}{} & \multicolumn{1}{c}{ Stat. } & Syst. (fit range) & Syst. (fit func.) \\
\hline$\beta=5.85$ & $f_{\pi}^{\mathrm{TP}}=0.0489$ & \pm 0.0056 & +0.0008 & -0.0017 & +0.0 & -0.0011 \\
& $f_{\pi}^{\mathrm{TP}}=94.1$ & \pm 11.8 & +1.6 & -3.3 & +0.0 & $-2.2 \mathrm{MeV}$ \\
$\beta=6.00$ & $f_{\pi}^{\mathrm{TP}}=0.0394$ & \pm 0.0027 & +0.0011 & -0.0 & +0.0 & -0.0013 \\
& $f_{\pi}^{\mathrm{TP}}=91.7$ & \pm 7.2 & +2.7 & -0.0 & +0.0 & $-3.0 \mathrm{MeV}$ \\
& $f_{\pi}^{\mathrm{MC}}=0.0367$ & \pm 0.0024 & +0.0011 & -0.0 & +0.0 & -0.0014 \\
& $f_{\pi}^{\mathrm{MC}}=85.4$ & \pm 6.4 & +2.5 & -0.0 & +0.0 & $-3.4 \mathrm{MeV}$
\end{tabular}

The values of $f_{\pi}$ obtained with the tadpole improved renormalization constants are consistent with the experimental value within the statistical errors (see Fig. 27). That with the MC renormalization constant is also consistent with experiment if we take account of the (small) systematic error.
However, we should take these numbers with caution, because $\chi^{2} / N_{\mathrm{DF}}$ for the extrapolation is not small enough, as mentioned above. Note that the decay constants in the chiral limit are consistent with the GF11 data [24], although the errors in our results are considerably larger.

TABLE XIX. Pion masses determined from $\widetilde{\pi}$ propagators.

\begin{tabular}{|c|c|c|c|c|c|c|c|c|c|c|c|}
\hline \multirow[b]{3}{*}{$K$} & \multicolumn{4}{|c|}{$\beta=5.85$} & \multicolumn{7}{|c|}{$\beta=6.0$} \\
\hline & \multicolumn{2}{|c|}{$t_{0}=t_{\min }=12$} & \multirow{2}{*}{\multicolumn{2}{|c|}{$\begin{array}{l}t_{0}=t_{\chi^{2}} \\
m_{\tilde{\pi}}\end{array}$}} & \multirow[b]{2}{*}{$\chi^{2} / N_{\mathrm{DF}}$} & \multirow[b]{2}{*}{$K$} & \multicolumn{2}{|c|}{$t_{0}=t_{\min }=15$} & \multirow[b]{2}{*}{$t_{\chi^{2}}$} & \multirow[t]{2}{*}{$t_{0}=t_{\chi^{2}}$} & \multirow[b]{2}{*}{$\chi^{2} / N_{\mathrm{DF}}$} \\
\hline & $m_{\widetilde{\pi}}$ & $\chi^{2} / N_{\mathrm{DF}}$ & & & & & $m_{\tilde{\pi}}$ & $\chi^{2} / N_{\mathrm{DF}}$ & & & \\
\hline 0.1440 & $1.0299(14)$ & 1.32 & 8 & 1.0304 & 1.68 & 0.1450 & $0.8059(9)$ & 0.49 & 12 & 0.8068 & 1.06 \\
\hline 0.1540 & $0.6106(21)$ & 0.96 & 7 & 0.6117 & 1.18 & 0.1520 & $0.4747(14)$ & 0.35 & 7 & 0.4767 & 0.91 \\
\hline 0.1585 & $0.3753(34)$ & 0.97 & 5 & 0.3774 & 1.41 & 0.1550 & $0.2937(24)$ & 0.88 & 6 & 0.2967 & 0.89 \\
\hline 0.1595 & $0.3070(42)$ & 1.00 & 4 & 0.3097 & 1.45 & 0.1555 & $0.2559(30)$ & 0.85 & 6 & 0.2593 & 0.83 \\
\hline 0.1605 & $0.2127(64)$ & 1.02 & 4 & 0.2175 & 1.31 & 0.1563 & $0.1804(66)$ & 0.68 & 5 & 0.1897 & 0.75 \\
\hline
\end{tabular}




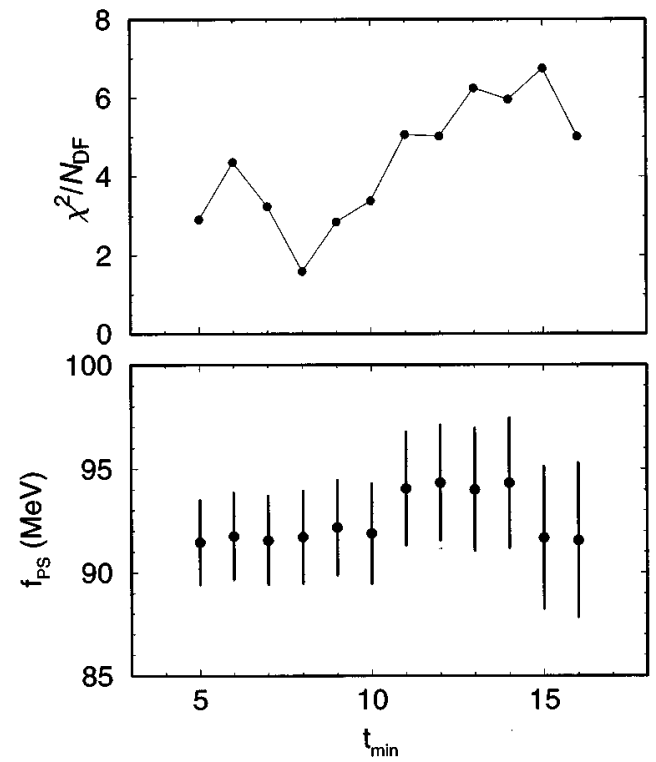

FIG. 30. Pseudoscalar meson decay constant at zero quark mass versus $t_{\min }$, together with $\chi^{2} / N_{\mathrm{DF}}$. The errors are estimated by the least mean square fit.

\section{CONCLUSIONS AND DISCUSSION}

In analyses of numerical simulations aiming toward high precision determination of light hadron masses, one first encounters the problem of the fitting range for hadron propagators. We find that effective masses of hadrons in general do not exhibit clear plateaus, although the statistics is relatively high [the number of configurations is 100 (200) at $\beta=5.85$ (6.0)]. The correlated $\chi^{2}$ fits do not determine unambiguously the time slice beyond which the ground state dominates. We also notice the very intriguing fact that $m_{\text {fit }}$ obtained by the correlated fits to a range from $t=t_{0}$ has a strong correlation with $m_{\text {eff }}$ at $t=t_{0}$. Varying the fitting range systematically, we estimate the systematic errors in hadron masses due to statistical fluctuations as well as due to the contamination from excited states, which cannot be properly taken into account by the standard least mean square fit with a fixed fitting range. We find that the systematic errors for the hadron masses with quarks lighter than the strange quark amount to 1-2 times the statistical errors.

When the lattice scale is fixed from the $\rho$ meson mass, the masses of the $\Omega^{-}$baryon and the $\phi$ meson at two $\beta$ 's agree with experiment within about one standard deviation. On the other hand, the central value of the nucleon mass at $\beta=6.0$
(5.85) is larger than its experimental value by about $15 \%$ (20\%) and that of the $\Delta$ mass by about $15 \%(4 \%)$ : Even when the systematic errors are included, the baryon masses at $\beta=6.0$ do not agree with experiment. In order to take the continuum limit of the nucleon mass and the $\Delta$ mass, we need data for a wider range of $\beta$ with statistical and systematic errors much reduced. For the masses of excited states of the $\rho$ meson and the nucleon, there exist two-mass fits which do not contradict experiment, except for the case of the nucleon at $\beta=6.0$. Although this does not necessarily imply that the excited state masses appear consistent with experiment because two-mass fits are very unstable, the existence of such a fit consistent with experiment encourages us to perform more work in this direction.

Determination of meson decay constants is usually accompanied by uncertainties of renormalization constants. One can in principle employ any renormalization constant such as that determined by naive perturbation theory or tadpole improved perturbation theory. We have indeed shown that when we use renormalization constants given by tadpole improved perturbation theory, although the decay constants for the $\phi, \rho, K$, and $\pi$ mesons are in general off experiment at finite lattice spacing, for example, by $30-40 \%$ at $m_{\rho} a=0.33-0.40$ in the case of the $F_{\phi}$, they approach in the continuum limit toward values consistent with the experimental values.

It is, however, desirable to employ a renormalization constant which gives weak $a$ dependence for the decay constants. We have shown that when we use the renormalization constants determined by Monte Carlo simulations, the vector meson decay constants at two $\beta$ 's agree remarkably with each other and reproduce the experimental values within the errors for a wide range of the quark mass with the chiral limit included. This implies a strong advantage to applying renormalization constants determined nonperturbatively. For pseudoscalar mesons, however, we find that although the decay constant $f_{P}^{\mathrm{MC}}$ in the chiral limit agrees with the experimental value of $f_{\pi}$, albeit with large errors, it differs from the experimental value of $f_{K}$ by about $20 \%$ at $m_{\rho} a=0.33$. This discrepancy might be due to systematic errors in the numerical calculation of $Z_{A}$. These results imply the importance of more systematic nonperturbative determination of the renormalization constants for various meson decays.

Note added. After this work was completed, three groups reported results of high statistics studies of the hadron spectrum [25-27] at $\beta=6.0$. Their results are consistent with ours.

TABLE XX. Pseudoscalar meson decay constants in lattice units. In parentheses are errors estimated by the jackknife method. Errors given in the form ${ }_{- \text {lower }}^{+ \text {upper }}$ are for the fitting range dependent upper (lower) bounds.

\begin{tabular}{lcccccc}
\hline \hline & \multicolumn{2}{c}{$\beta=5.85$} & & & \multicolumn{2}{c}{$\beta=6.0$} \\
$K$ & $f_{P}^{\mathrm{PT}}$ & $f_{P}^{\mathrm{TP}}$ & $K$ & $f_{P}^{\mathrm{PT}}$ & $f_{P}^{\mathrm{TP}}$ & $f_{P}^{\mathrm{MC}}$ \\
\hline 0.1440 & 0.1152 & $0.1443(25)_{-5}^{+12}$ & 0.1450 & 0.0892 & $0.1030(10)_{-0}^{+12}$ & 0.0710 \\
0.1540 & 0.0922 & $0.0929(22)_{-1}^{+10}$ & 0.1520 & 0.0713 & $0.0701(11)_{-0}^{+18}$ & 0.0567 \\
0.1585 & 0.0732 & $0.0664(26)_{-14}^{+12}$ & 0.1550 & 0.0566 & $0.0517(13)_{-10}^{+16}$ & 0.0450 \\
0.1595 & 0.0677 & $0.0600(30)_{-15}^{+19}$ & 0.1555 & 0.0535 & $0.0482(15)_{-15}^{+15}$ & 0.0426 \\
0.1605 & 0.0597 & $0.0515(33)_{-9}^{+32}$ & 0.1563 & 0.0462 & $0.0408(26)_{-23}^{+30}$ & 0.0368 \\
\hline \hline
\end{tabular}




\section{ACKNOWLEDGMENTS}

Numerical simulations were performed under the QCDPAX project which is supported by Grants-in-Aid of the
Ministry of Education, Science and Culture (Nos. 62060001 and 02402003). Analyses of data were also supported in part by Grants-in-Aid of the Ministry of Education, Science and Culture (Nos. 07NP0401, 07640375, and 07640376).
[1] Y. Iwasaki and T. Yoshié, Phys. Lett. B 216, 387 (1989); Y. Iwasaki, in Lattice '88, Proceedings of the International Symposium, Batavia, Illinois, edited by A. S. Kronfield and P. B. Mackenzie [Nucl. Phys. B (Proc. Suppl.) 9, 254 (1989)].

[2] T. Yoshié, Y. Iwasaki, and S. Sakai, in Lattice '89, Proceedings of the International Symposium, Capri, Italy, edited by R. Petronzio et al. [Nucl. Phys. B (Proc. Suppl.) 17, 413 (1990)].

[3] APE Collaboration, P. Bacilieri et al., Phys. Lett. B 214, 115 (1988); Nucl. Phys. B317, 509 (1989); APE Collaboration, S. Cabasino et al., in Lattice '89 [2], p. 431.

[4] QCDPAX Collaboration, Y. Iwasaki et al., in Lattice '92, Proceedings of the International Symposium, Amsterdam, The Netherlands, edited by J. Smit and P. van Baal [Nucl. Phys. B (Proc. Suppl.) 30, 397 (1993)].

[5] QCDPAX Collaboration, Y. Iwasaki et al., in Lattice '93, Proceedings of the International Symposium, Dallas, Texas, edited by T. Draper et al. [Nucl. Phys. B (Proc. Suppl.) 34, 354 (1994)].

[6] APE Collaboration, C. R. Allton et al., in Lattice '93 [5], p. 360.

[7] APE Collaboration, S. Cabasino et al., Phys. Lett. B 258, 195 (1991).

[8] APE Collaboration, M. Guagnelli et al., Nucl. Phys. B378, 616 (1992).

[9] K. M. Bitar et al., Phys. Rev. D 46, 2169 (1992).

[10] F. Butler, H. Chen, J. Sexton, A. Vaccarino, and D. Weingarten, Nucl. Phys. B430, 179 (1994).

[11] T. Bhattacharya and R. Gupta, in Lattice '94, Proceedings of the International Symposium, Bielefeld, Germany, edited by F. Karsch et al. [Nucl. Phys. B (Proc. Suppl.) 42, 935 (1995)].

[12] UKQCD Collaboration, C. R. Allton et al., Phys. Rev. D 49, 474 (1994).
[13] D. Daniel et al., Phys. Rev. D 46, 3130 (1992).

[14] Y. Iwasaki et al., Comput. Phys. Commun. 49, 449 (1988); T. Shirakawa et al., in Proceedings of Supercomputing '89, Reno, Nevada, 1989 (unpublished), p. 495; Y. Iwasaki et al., in Lattice '89 [2], p. 259.

[15] UKQCD Collaboration, C. R. Allton et al., Phys. Rev. D 47, 5128 (1993).

[16] UKQCD Collaboration, P. Lacock and C. Michael, Phys. Rev. D 52, 5213 (1995).

[17] S. Sharpe, Phys. Rev. D 41, 3233 (1990); 46, 3146 (1992).

[18] C. Bernard and M. Golterman, Phys. Rev. D 46, 853 (1992).

[19] G. Martinelli and Y. C. Zhang, Phys. Lett. 123B, 433 (1983).

[20] G. P. Lepage, in Lattice '91, Proceedings of the International Symposium, Tsukuba, Japan, edited by M. Fukugita et al. [Nucl. Phys. B (Proc. Suppl.) 26, 45 (1992)]; A. S. Kronfeld, in Lattice '92 [4], p. 445; P. B. Mackenzie, ibid., p. 35.

[21] G. P. Lepage and P. B. Mackenzie, Phys. Rev. D 48, 2250 (1993).

[22] A. X. El-Khadra et al., Phys. Rev. Lett. 69, 729 (1992).

[23] L. Maiani and G. Martinelli, Phys. Lett. B 178, 265 (1986).

[24] F. Butler, H. Chen, J. Sexton, A. Vaccarino, and D. Weingarten, Nucl. Phys. B421, 217 (1994).

[25] JLQCD Collaboration, S. Aoki et al., Lattice '95, Proceedings of the International Symposium, Melbourne, Australia (unpublished), Tsukuba Report Nos. UTHEP-323, hep-lat/9510013 (unpublished).

[26] M. Göckeler et al., Phys. Rev. D 53, 2317 (1996).

[27] T. Bhattacharya, R. Gupta, G. Kilcup, and S. Sharpe, this issue, Phys. Rev. D 53, 6486 (1996); T. Bhattacharya and R. Gupta, ibid. (to be published).

[28] S. Ono, Phys. Rev. D 17, 888 (1978). 